\title{
ON THE CHOW MOTIVE OF SOME MODULI SPACES.
}

\author{
SEBASTIAN DEL BAÑO
}

\begin{abstract}
We study the motive of moduli spaces of stable vector bundles over a smooth projective curve. We prove this motive lies in the category generated by the motive of the curve and we compute its class in the Grothendieck ring of the category of motives. As applications we compute the Poincaré-Hodge polynomials and the number of points over a finite field and we study some conjectures on algebraic cycles on these moduli spaces.
\end{abstract}

\section{INTRODUCTION}

The cohomology of the moduli spaces of stable vector bundles over a smooth projective curve has been thoroughly studied over the last years. This study has been accomplished by topological methods involving the Narasimhan-Seshadri correspondence ([34]), by number theoretical methods ([22], [23]) and using differential geometry ([1]).

The work we present is a generalisation of some of these results to the more general setup of motives. We use a geometric construction due to E. Bifet, M. Letizia and F. Ghione to compute the motivic Poincaré polynomial of these moduli spaces and show that its motive lies in the category of motives generated by the motive of the curve. As corollaries we produce a closed formula for the Poincaré-Hodge polynomial and the number of points over a finite field of these varieties. We also study some well known conjectures for these moduli spaces, namely the Hodge, Tate and standard conjectures.

We have obtained a number of intermediate results which are of independent interest: A localisation theorem for actions of the multiplicative group and a study of the symmetric powers of a motive. Similar results have been obtained by E. Getzler in [17], quite surprisingly in studying a problem similar to ours: the motive of the moduli space of (pointed) curves.

Acknowledgements. I wish to express my gratitude to Emili Bifet, Vicente Navarro Aznar and Pere Pascual Gainza for many helpful discussions. I also thank the Department of Mathematics at the State University of New York at Stony Brook where parts of this work were carried out.

Notations and Conventions. The Poincaré and Poincaré-Hodge polynomial of a variety $X$ will be denoted by $P_{t}(X)$ and $P_{x y}(X)$ respectively. $X^{(n)}$ will stand for the $n$-th symmetric power of a variety $X$, that is the quotient of $X^{n}$ by the natural action of the symmetric group on it. We shall adopt the notations of Scholl ([36]) for the theory of motives. If $X$ is an object in an additive category we write $[X]$ for its class in the Grothendieck group. When it is clear from the context, we shall sometimes drop the [ ] and note in the same way the object and its class.

From section 4 onwards we use the theory of [21], therefore we assume that the characteristic of the base field is zero unless otherwise indicated.

Date: October 18, 1998.

1991 Mathematics Subject Classification. 14C25, 14D20.

Key words and phrases. Motives, moduli spaces, vector bundles.

Partially supported by DGCYT grant PB93-0790. 


\section{Preliminaries}

\subsection{Motives.}

1.1.1. Definitions. Let $k$ be a field. The category of smooth projective varieties over $k$ will be noted by $\mathcal{V}_{k}$. The theory of Chow motives (see [30] or [36]) provides a functor $h: \mathcal{V}_{k} \longrightarrow \mathcal{M}_{k}^{+}$ where $\mathcal{M}_{k}^{+}$is a $\mathbb{Q}$-linear pseudoabelian tensor category called the category of effective motives. For each $X \in \mathrm{Ob}\left(\mathcal{V}_{k}\right)$ the object $h(X)$ is called the motive of $X$. The class of this object in the Grothendieck ring $K_{0}\left(\mathcal{M}_{k}^{+}\right)$will be noted by $\chi(X)$ and called the motivic Poincaré polynomial.

1.1.2. Motives for arbitrary schemes. The functor $h$ has recently been extended to the category, $\mathbf{S c h}_{k}$, of arbitrary separated schemes of finite type over a field of characteristic zero $k$ by $\mathrm{F}$. Guillén and V. Navarro ([21]). This extension now takes values in a category of bounded complexes of effective motives up to homotopy equivalence, $h: \mathbf{S c h}_{k} \longrightarrow$ Ho $C^{b} \mathcal{M}_{k}^{+}$. An essential rôle in this extension theorem is played by the simple functor which associates an object of $C^{b} \mathcal{M}_{k}^{+}$to each cubical diagram in $C^{b} \mathcal{M}_{k}^{+}$, as in [21] we shall note this functor by $\mathbf{s}$. The simplest nontrivial example of cubical diagram is a morphism, in this case the functor $\mathbf{s}$ coincides with the cone.

By taking the class in the Grothendieck ring we obtain an extension of the motivic Poincaré polynomial to arbitrary schemes: $\chi: \mathrm{Ob}\left(\mathbf{S c h}_{k}\right) \longrightarrow K_{0}\left(\mathcal{M}_{k}^{+}\right)$.

There is also a theory with compact supports giving rise to a functor from the category of schemes with proper morphisms $h_{c}: \mathbf{S c h}_{c, k} \longrightarrow \mathrm{Ho} C^{b} \mathcal{M}_{k}^{+}$. It is characterised by the following property: For any closed immersion $i: Y \hookrightarrow X$ we have an homotopy equivalence $h_{c}(X-Y) \simeq \mathbf{s}\left(i^{*}: h_{c} X \longrightarrow h_{c} Y\right)$.

Again by taking classes in $K_{0} \mathcal{M}_{k}^{+}$we obtain a morphism $\chi_{c}: \mathrm{Ob}\left(\mathbf{S c h}_{k}\right) \longrightarrow K_{0}\left(\mathcal{M}_{k}^{+}\right)$.

1.1.3. Realisations. A cohomology functor is defined to be a functor $\mathcal{V}_{k} \longrightarrow \mathcal{C}$ to a graded tannakian category such that composed with a fibre functor of $\mathcal{C}$ yields a Weil cohomology. The cohomology functors we will use are: singular cohomology taking values on the category of rational pure Hodge structures $\mathcal{V}_{k} \longrightarrow \mathbf{P} \mathbf{H S}_{\mathbb{Q}}$ and $\ell$-adic cohomology taking values in the category of graded $\ell$-adic representations of the Galois group $\operatorname{Gal}(\bar{k} \mid k) \mathcal{V}_{k} \longrightarrow \mathbf{G r}_{\mathbf{R e p}} \operatorname{Ral}(\bar{k} \mid k)$.

A cohomology functor canonically gives rise to an additive functor defined on $\mathcal{M}_{k}^{+}$. We call this extension a realisation functor. Observe that a realisation functor $\mathcal{M}_{k}^{+} \longrightarrow \mathcal{C}$ actually maps to the full subcategory of non-negative degree objects, we shall note this category by $\mathcal{C}^{+}$.

A realisation functor $\mathcal{M}_{k}^{+} \longrightarrow \mathcal{C}^{+}$gives rise to a ring morphism $K_{0}\left(\mathcal{M}_{k}^{+}\right) \longrightarrow K_{0}\left(\mathcal{C}^{+}\right)$. For instance, singular cohomology with complex coefficients defines a ring morphism $K_{0}\left(\mathcal{M}_{k}^{+}\right) \longrightarrow$ $K_{0}\left(\mathbf{P H S}_{\mathbb{C}}^{+}\right)=\mathbb{Z}[x, y]$, noted $P_{x y}$, which extends the Poincaré-Hodge polynomial. Over a finite field, $\mathbb{F}_{q}, \ell$-adic cohomology defines a ring morphism $K_{0}\left(\mathcal{M}_{k}^{+}\right) \longrightarrow K_{0}\left(\mathbf{G r}-\mathbf{R e p}_{\mathbb{Q}_{\ell}} G a l(\bar{k} \mid k)^{+}\right)$. Composing this morphism with the trace of Frobenius, $\left[\oplus V_{i}\right] \mapsto \sum \operatorname{Tr}\left(\left.\operatorname{Fr}\right|_{V^{i}}\right) \cdot(-t)^{i}$, we obtain a ring morphism $\nu_{q}^{t}: K_{0}\left(\mathcal{M}_{k}^{+}\right) \longrightarrow \mathbb{Q}_{\ell}[t]$. The trace formula shows that $\nu_{q}^{1}(\chi(X))=\# X\left(\mathbb{F}_{q}\right)$.

1.1.4. The ring $\mathcal{K}$. We define $\mathcal{K}$ to be the completion of $K_{0} \mathcal{M}_{k}^{+}$along the ideal generated by the Lefschetz motive. Given that $P_{x y}(\mathbb{L})=x y$ we see that $P_{x y}$ defines a ring morphism $\mathcal{K} \longrightarrow \mathbb{Z}[[x, y]]$ which we still note $P_{x y}$. Also, as $\nu_{q}^{t}(\mathbb{L})=q t^{2}, \nu_{q}^{t}$ extends to a ring morphism $\nu_{q}^{t}: \mathcal{K} \longrightarrow \mathbb{Q}_{\ell}[[t]]$.

Given an ind-scheme $\left(X_{\mu}\right)_{\mu \in M}$, we say that its motivic Poincaré polynomial stabilises if for each $n \in \mathbb{N}$ we can find a $\mu_{n} \in M$ such that for all $\mu>\mu_{n}$ we have $\chi\left(X_{\mu}\right)-\chi\left(X_{\mu_{n}}\right) \in\left(\mathbb{L}^{n}\right)$. In this case $\left(\chi\left(X_{\mu}\right)\right)_{\mu \in M}$ converges to an element of $\mathcal{K}$ that we call $\chi(X)$. 
1.2. Moduli spaces. Let $C$ be a smooth projective curve of genus $g>1$ over a field $k$. We shall note by $N_{C}(n, d)$ the moduli space of stable bundles of rank $n$ and degree $d$ over the curve $C$. If $(n, d)=1$ this is a smooth projective variety over $k$.

The construction of these moduli spaces can be extended to smooth projective curves over the spectrum of a discrete valuation ring.

Theorem 1.1. (Seshadri) Let $S$ be the spectrum of a discrete valuation ring with residue field $k$. Let $\mathcal{C}$ be a smooth projective curve over $S$ with special fibre $C$, and coprime integers $n>1$ and $d$. There exists a smooth projective scheme $N_{\mathcal{C}}(n, d)$ over $S$ whose generic fibre is the corresponding moduli space over the generic fibre of $\mathcal{C}$ and a natural morphism $N_{\mathcal{C}}(n, d) \times{ }_{S} \operatorname{Spec}(k) \longrightarrow$ $N_{C}(n, d)$ that induces a bijection on the set of $k$-points.

Proof. The proof runs along the lines of the corresponding proof over a field (see [33]) combined with the results of Seshadri on Geometric Invariant Quotients over an arbitrary base (see appendix $1 \mathrm{G}$ in [31]).

\section{Localisation of $\mathbb{G}_{m}$-ACTiOns}

The purpose of this section is prove a localisation theorem relating the Chow groups of a variety acted on by $\mathbb{G}_{m}$ to the Chow groups of the fixed components. We obtain a similar result concerning the Chow motive and algebraic $K$-theory. In the case of the fixed components being points this action yields a decomposition in cells and the results are well known: Grothendieck determined the Chow groups ([18]) and in the particular case of projective varieties homogeneous under reductive groups, Köck ([26]) has found an expression for the Chow motive.

We start by briefly recalling some facts on $\mathbb{G}_{m}$-actions.

2.1. $\mathbb{G}_{m}$-actions. Let $X$ be a smooth complete variety acted on by the multiplicative group and let $X^{\mathbb{G}_{m}}$ be the closed subscheme of fixed points. By a result of B. Iversen $X^{\mathbb{G}_{m}}$ is smooth ([24]). Let $X^{\mathbb{G}_{m}}=\sqcup_{\alpha} X_{\alpha}$ be its decomposition in connected components. Białinicki-Birula has proved the following theorem:

Theorem 2.1. ([2], Theorem 4.3) There exists a unique decomposition in $\mathbb{G}_{m}$-invariant locally closed subvarieties

$$
X=\bigsqcup_{\alpha} X_{\alpha}^{+}
$$

and $\mathbb{G}_{m}$-equivariant morphisms $\pi_{\alpha}: X_{\alpha}^{+} \longrightarrow X_{\alpha}$ such that

1. $\left(X_{\alpha}^{+}\right)^{\mathbb{G}_{m}}=X_{\alpha}$.

2. $\pi_{\alpha}$ is an affine fibration.

3. The inclusion $X_{\alpha}^{+} \subset X$ identifies $\left.T X_{\alpha}^{+}\right|_{X_{\alpha}}$ with the subbundle of $\left.T X\right|_{X_{\alpha}}$ on which $\mathbb{G}_{m}$ acts with positive weights.

In the projective case we have the following important result.

Theorem 2.2. ([3]) If $X$ is a smooth projective variety acted on by $\mathbb{G}_{m}$ then decomposition (1) is filtrable, that is there exists a sequence of $\mathbb{G}_{m}$-invariant closed subvarieties

$$
X=Y_{0} \supset Y_{1} \supset \cdots \supset Y_{n} \supset Y_{n+1}=\emptyset
$$

with $Y_{\alpha}-Y_{\alpha+1}=X_{\alpha}^{+}$. 
2.2. Localisation. We shall use the higher Chow groups as defined by Bloch in [9]. For each variety $X$ and non-negative integers $i$ and $j$ we have a group $C H^{i}(X, j)$. For $j=0$ these coincide with the ordinary Chow groups.

Let $X$ be a smooth projective variety over a field $k$ acted on by the multiplicative group $\mathbb{G}_{m}$. Let $\left\{X_{\alpha}\right\}_{\alpha},\left\{X_{\alpha}^{+}\right\}_{\alpha}$ and $\pi_{\alpha}$ be as in the previous subsection.

Definition 2.3. Define $d_{\alpha}$ to be the codimension of $X_{\alpha}^{+}$in $X$. Let $\Gamma_{\pi_{\alpha}}$ be the graph of $\pi_{\alpha}$ : $X_{\alpha}^{+} \longrightarrow X_{\alpha}$ and $m_{\alpha}$ the class in

$$
C H^{\operatorname{dim} X_{\alpha}+d_{\alpha}}\left(X_{\alpha} \times X\right)
$$

of its closure in $X_{\alpha} \times X$.

The correspondence $m_{\alpha}$ defines morphisms

$$
C H^{i-d_{\alpha}}\left(X_{\alpha}, j\right) \stackrel{p_{1}^{*}}{\longrightarrow} C H^{i-d_{\alpha}}\left(X_{\alpha} \times X, j\right) \stackrel{\cdot \cap m_{\alpha}}{\longrightarrow}
$$

$$
C H^{i+\operatorname{dim} X_{\alpha}}\left(X_{\alpha} \times X, j\right) \stackrel{p_{2 *}}{\longrightarrow} C H^{i}(X, j)
$$

which we still note by $m_{\alpha}$. Note that this is well defined: $p_{1}^{*}$ is defined as $p_{1}$ is flat ([9] Proposition 1.3), $p_{2 *}$ is defined because $p_{2}$ is proper and finally $\cdot \cap m_{\alpha}$ is defined by Theorem 4.1 in [9] given the fact that $X_{\alpha} \times X$ is smooth.

Theorem 2.4. If $X$ is projective then the $m_{\alpha}$ define isomorphisms of higher Chow groups

$$
\bigoplus_{\alpha} C H^{i-d_{\alpha}}\left(X_{\alpha}, j\right) \stackrel{\simeq}{\longrightarrow} C H^{i}(X, j)
$$

of algebraic K-theory groups

$$
\bigoplus_{\alpha} K^{i}\left(X_{\alpha}\right) \stackrel{\simeq}{\longrightarrow} K^{i}(X)
$$

and Chow motives

$$
\bigoplus_{\alpha} h X_{\alpha}\left(-d_{\alpha}\right) \longrightarrow h X
$$

Proof. Given that $X$ is projective we have a filtration as in theorem 2.2, we use the same notations as in that theorem.

We first prove (2). This is achieved by splitting the localization exact sequence for the higher Chow groups of the closed immersion $i_{\alpha}: Y_{\alpha+1} \hookrightarrow Y_{\alpha}$. Let $j_{\alpha}$ be the complementary open immersion $X_{\alpha}^{+} \hookrightarrow Y_{\alpha}$ and $e_{\alpha}=\operatorname{codim}_{Y_{\alpha}} Y_{\alpha+1}$. Then by the localization theorem ([9] Theorem 3.1 , corrected in [10] Corollary 0.2) we have an exact sequence

$$
\begin{aligned}
\cdots \stackrel{i_{\alpha *}}{\longrightarrow} C H^{i-e_{\alpha}}\left(Y_{\alpha+1}, 1\right) \stackrel{j_{\alpha}^{*}}{\longrightarrow} C H^{i}\left(X_{\alpha}^{+}, 1\right) \stackrel{\partial}{\longrightarrow} \\
\quad \stackrel{\partial}{\longrightarrow} C H^{i-e_{\alpha}}\left(Y_{\alpha+1}, 0\right) \stackrel{i_{\alpha *}}{\longrightarrow} C H^{i}\left(Y_{\alpha}, 0\right) \stackrel{j_{\alpha}^{*}}{\longrightarrow} C H^{i}\left(X_{\alpha}^{+}, 0\right) \longrightarrow 0 .
\end{aligned}
$$

By the homotopy principle (which is proven for affine fibrations in the same way as for vector bundles, see [9] Corollary 3.7) the projection $\pi_{\alpha}: X_{\alpha}^{+} \longrightarrow X_{\alpha}$ induces an isomorphism

$$
\pi_{\alpha}^{*}: C H^{i}\left(X_{\alpha}^{+}, j\right) \longrightarrow C H^{i}\left(X_{\alpha}, j\right) .
$$


Let $\bar{\Gamma}_{\pi_{\alpha}}$ be the closure in $Y_{\alpha} \times X_{\alpha}$ of $\Gamma_{\pi_{\alpha}}$. We have the projections

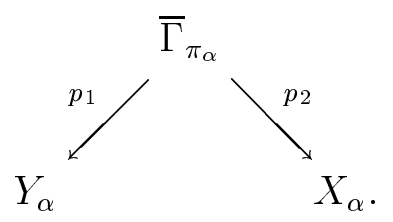

As $X_{\alpha}$ is smooth, by [9] Theorem 4.1, $p_{2}$ induces a morphism $p_{2}^{*}: C H^{i}\left(X_{\alpha}, j\right) \longrightarrow C H^{i}\left(\bar{\Gamma}_{\pi_{\alpha}}, j\right)$ and $p_{1}$ being proper induces $p_{1 *}: C H^{i}\left(\bar{\Gamma}_{\pi_{\alpha}}, j\right) \longrightarrow C H^{i}\left(Y_{\alpha}, j\right)$. Let $\gamma_{\alpha}=p_{1 *} p_{2}^{*}$, we claim that $\gamma_{\alpha}\left(\pi_{\alpha}^{*}\right)^{-1}$ is a section of $j_{\alpha}^{*}$. This provides a splitting of the exact sequence (5).

To prove the claim consider the flat base change

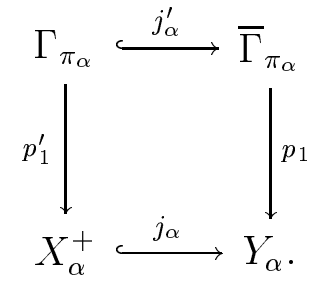

Then according to lemma 2.5 we obtain

$$
j_{\alpha}^{*} \gamma_{\alpha}\left(\pi_{\alpha}^{*}\right)^{-1}=j_{\alpha}^{*} p_{1 *} p_{2}^{*}\left(\pi_{\alpha}^{*}\right)^{-1}=p_{1 *}^{\prime} j_{\alpha}^{\prime *} p_{2}^{*}\left(\pi_{\alpha}^{*}\right)^{-1}=\pi_{\alpha}^{*}\left(\pi_{\alpha}^{*}\right)^{-1}=I d .
$$

This proves the claim.

Now if we put together the split exact sequences (5) from $\alpha=0$ to $n$ we get

$$
\begin{array}{rccc}
0 \longrightarrow C H^{i-d_{1}}\left(Y_{1}, j\right) \stackrel{i_{0 *}}{\longrightarrow} C H^{i}(X, j) & \stackrel{j_{0}^{*}}{\longrightarrow} C H^{i}\left(X_{0}^{+}, j\right) \stackrel{\pi_{0}^{*}}{\simeq} C H^{i}\left(X_{0}, j\right) \longrightarrow 0 \\
0 \longrightarrow C H^{i-d_{2}}\left(Y_{2}, j\right) \stackrel{i_{1 *}}{\longrightarrow} C H^{i-d_{1}}\left(Y_{1}, j\right) & \stackrel{j_{1}^{*}}{\longrightarrow} C H^{i-d_{1}}\left(X_{1}^{+}, j\right) \stackrel{\pi_{1}^{*}}{\longrightarrow} C H^{i-d_{1}}\left(X_{1}, j\right) \longrightarrow 0 \\
\ldots & \ldots & \ldots & \ldots \\
0 & \ldots H^{i-d_{n}}\left(Y_{n}, j\right) & \stackrel{j_{n}^{*}}{\longrightarrow} C H^{i-d_{n}}\left(X_{n}^{+}, j\right) \stackrel{\pi_{n}^{*}}{\simeq} C H^{i-d_{n}}\left(X_{n}, j\right) \longrightarrow 0
\end{array}
$$

and adding up all the splittings we have constructed we obtain a natural isomorphism

$$
\bigoplus_{\alpha=0}^{n} C H^{i-d_{\alpha}}\left(X_{\alpha}, j\right) \stackrel{\oplus i_{0 *} i_{1 *} \cdots i_{\alpha-1 *} \gamma_{\alpha}}{\longrightarrow} C H^{i}(X, j) \text {. }
$$

Next note that $i_{0 *} i_{1 *} \cdots i_{\alpha-1 *} \gamma_{\alpha}$ is the morphism induced by $\bar{\Gamma}_{\pi_{\alpha}} \subset X \times X_{\alpha}$ (as in the definition of $\gamma_{\alpha}$ above). To see that this coincides with $m_{\alpha}$ it is enough to see that the following diagram

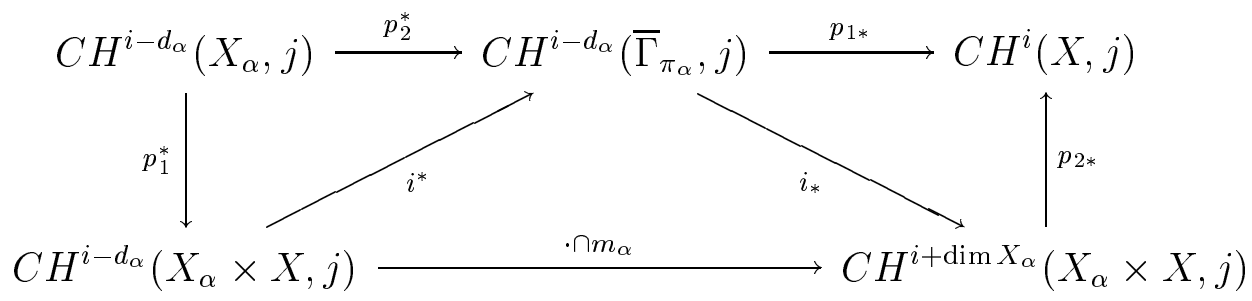

commutes, where $p_{1}$ and $p_{2}$ are in each case the natural projections and $i$ is the closed immersion $\bar{\Gamma}_{\pi_{\alpha}} \subset X_{\alpha} \times X$. The two top triangles commute by functoriality, the lower one commutes by [9], 5.8.i. This proves (2).

For (4) note that the isomorphism $\oplus_{\alpha} C H^{i-d_{\alpha}} X_{\alpha} \stackrel{\simeq}{\longrightarrow} C H^{i} X$ is induced by the correspondence $m_{\alpha}$. If $S$ is a smooth projective variety and we replace $X$ by $X \times S$ there is still a $\mathbb{G}_{m}$-action 
with fixed point set $\sqcup X_{\alpha} \times S$, the previous arguments show that the correspondences $m_{\alpha} \times S$ induce an isomorphism $\oplus_{\alpha} C H^{i-d_{\alpha}}\left(X_{\alpha} \times S\right) \stackrel{\simeq}{\longrightarrow} C H^{i}(X \times S)$. Manin's identity principle ([30], see also [36], 2.3) yields the result.

The statement for the algebraic $K$-theory groups is proved in the same fashion using the corresponding properties of $K$-theory in [35].

Lemma 2.5. Let $X, U$ and $X^{\prime}$ be varieties and consider a flat morphism $j: U \longrightarrow X$ and a proper morphism $f: X^{\prime} \longrightarrow X$ form the cartesian diagram,

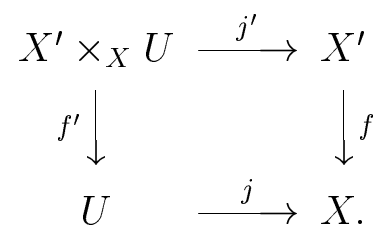

Then $j^{*} f_{*}=f_{*}^{\prime} j^{\prime *}$ in the higher Chow groups.

Proof. This results from the definition of the higher Chow groups in [9] together with the fact that the equality $j^{*} f_{*}=f_{*}^{\prime} j^{\prime *}$ holds already for the group of cycles on $X^{\prime}$ (Proposition 1.7 in $[16])$.

\section{3. $\lambda$-Structure in $\mathcal{M}_{\mathbf{k}}$}

In this section we introduce the notion of a $\lambda$-structure on a tensor category and show that each $\mathbb{Q}$-linear pseudoabelian tensor category has a canonical $\lambda$-structure. A consequence of this is that the wedge of a motive as defined in [27] verifies the fundamental property 3.1.3. However we prefer the notation $\lambda^{n}$ to $\wedge^{n}$ as for instance one has $\lambda^{n} \mathbb{L}=\mathbb{L}^{n}$. We shall use this to generalise a theorem of S̆hermenev and a formula of MacDonald.

\section{1. $\lambda$-structures.}

Definition 3.1. A $\lambda$-structure on an additive tensorial category $\mathcal{C}$ is a sequence of functors

$$
\lambda_{n}: \mathcal{C} \longrightarrow \mathcal{C}, \quad n \in \mathbb{N}
$$

such that:

1. $\lambda_{0}$ is the functor that takes all objects to $\mathbb{1}$ and all morphisms to the identity.

2. $\lambda_{1}$ is the identity functor.

3. $\lambda_{n}(X \oplus Y)$ is naturally isomorphic to $\oplus_{a+b=n} \lambda_{a}(X) \otimes \lambda_{b}(Y)$.

Remark 3.2. Given an additive tensor category, $\mathcal{C}$, with a $\lambda$-structure. Property 3 in definition 3.1 shows that the function

$$
\begin{aligned}
\lambda_{t}: \mathrm{Ob} \mathcal{C} & \longrightarrow K_{0} \mathcal{C}[[t]]^{*} \\
M & \mapsto \sum_{i}\left[\lambda^{i}(M)\right] \cdot t^{i}
\end{aligned}
$$

is additive, i.e. $\lambda_{t}(M \oplus N)=\lambda_{t}(M) \cdot \lambda_{t}(N)$. Therefore it defines a morphism of groups $\lambda_{t}: K_{0} \mathcal{C} \longrightarrow K_{0} \mathcal{C}[[t]]^{*}$. Properties 1 and 2 in definition 3.1 show that this makes $K_{0} \mathcal{C}$ a $\lambda$-ring. 
3.2. $\lambda$-structure on a pseudoabelian tensor category. Let $\mathcal{C}$ be a $\mathbb{Q}$-linear pseudoabelian tensor category. Whenever $p \in \operatorname{End}(M)$ is a projector we shall use the notation $(M, p)$ for the image of $p$.

The tensor category, $\mathcal{C}$, comes equipped with commutation constraints ([13]), that is an isomorphism $\varphi_{M N}: M \otimes N \longrightarrow N \otimes M$ for every pair of objects $M$ and $N$. In Proposition 1.5 in loc.cit. these commutation constraints are extended to more than one factor. In particular for $M \in \mathrm{Ob} \mathcal{C}$ they give a morphism of groups

$$
\begin{aligned}
\varphi: \mathfrak{S}_{n} & \longrightarrow \operatorname{Aut}_{\mathcal{C}}\left(M^{\otimes n}\right) \\
\sigma & \mapsto \varphi(\sigma) .
\end{aligned}
$$

It is readily checked that $\sum_{\sigma \in \mathfrak{S}_{n}} \varphi(\sigma)$ defines a projector in the $\mathbb{Q}$-algebra $\operatorname{End}_{\mathcal{C}}\left(M^{\otimes n}\right)$.

Definition 3.3. (c.f. [27]) For each $M \in \mathrm{Ob} \mathcal{C}$ and $n \in \mathbb{N}$ set

$$
\lambda^{n}(M)=\left(M^{\otimes n}, \frac{1}{n !} \sum_{\sigma \in \mathfrak{S}_{n}} \varphi(\sigma)\right)
$$

(this makes sense since $\mathcal{C}$ is pseudoabelian). For $f \in \operatorname{Hom}_{\mathcal{C}}(M, N)$ set

$$
\lambda^{n}(f)=\frac{1}{n !} \sum_{\sigma \in \mathfrak{S}_{n}} \varphi(\sigma) \circ f \otimes \cdots \otimes f \circ \frac{1}{n !} \sum_{\sigma \in \mathfrak{S}_{n}} \varphi(\sigma) \in \operatorname{Hom}_{\mathcal{C}}\left(\lambda^{n}(M), \lambda^{n}(N)\right) .
$$

This defines a functor $\lambda^{n}: \mathcal{C} \longrightarrow \mathcal{C}$ for each $n \in \mathbb{N}$.

Now we come to the main result of this section.

Theorem 3.4. The functors $\lambda^{n}$ define a $\lambda$-structure on the category $\mathcal{C}$.

Proof. Note that 3.1.1 and 3.1.2 are obvious from definition 3.3. The proof of 3.1.3 is more intricate.

The one thing we need to prove is $\lambda^{n}(M \oplus N) \simeq \bigoplus_{a+b=n} \lambda^{a} M \otimes \lambda^{b} N$. Write $X=M \oplus N$ and let $p \in \operatorname{End}_{\mathcal{C}} X$ be the projection on $M$ so that $M=(X, p)$ and $N=(X, 1-p)$.

Throughout the proof we shall simplify the notation $\varphi_{\sigma}$ and write $\sigma$ for the morphism induced by $\sigma \in \mathfrak{S}_{n}$.

We are concerned with

$$
\lambda^{n}(X)=\left(X^{\otimes n}, \frac{1}{n !} \sum_{\sigma \in \mathfrak{S}_{n}} \sigma\right)
$$

write $I d_{X \otimes n}$ as a sum of orthogonal projectors

$$
I d_{X \otimes n}=(p+(1-p))^{\otimes n}=\sum_{a+b=n} \begin{gathered}
\text { products of } a p^{\prime} s \\
\text { and } b(1-p)^{\prime} s
\end{gathered},
$$

in order to write this expression more conveniently choose a set of representatives $\sigma_{1}, \ldots, \sigma_{r}$ of $\mathfrak{S}_{n} /\left(\mathfrak{S}_{a} \times \mathfrak{S}_{b}\right)$, then

$$
I d_{X \otimes n}=\sum_{a+b=n} \sum_{i} \sigma_{i} p^{a}(1-p)^{b} \sigma_{i}^{-1}
$$

We claim that for fixed $a$ and $b, \mathfrak{S}_{n}$ leaves the projector

$$
\sum_{i} \sigma_{i} p^{a}(1-p)^{b} \sigma_{i}^{-1}
$$


invariant. To prove the claim note that for each $\sigma \in \mathfrak{S}_{n}$ the element $\sigma \sigma_{i}$ can be uniquely written in the form $\sigma_{i^{\prime}} h$ with $h \in \mathfrak{S}_{a} \times \mathfrak{S}_{b}$, in fact $i \mapsto i^{\prime}$ is a bijection. Then as $h$ commutes with $p^{a}(1-p)^{b}$

$$
\begin{aligned}
\sigma \sum_{i} \sigma_{i} p^{a}(1-p)^{b} \sigma_{i}^{-1} & =\sum_{i} \sigma_{i^{\prime}} h p^{a}(1-p)^{b} \sigma_{i}^{-1} \\
& =\sum_{i} \sigma_{i^{\prime}} p^{a}(1-p)^{b} h \sigma_{i}^{-1}=\sum_{i^{\prime}} \sigma_{i^{\prime}} p^{a}(1-p)^{b} \sigma_{i^{\prime}}^{-1} \sigma .
\end{aligned}
$$

This proves that $(6)$ is $\mathfrak{S}_{n}$-invariant.

We have the decomposition

$$
\lambda^{n}(X)=\bigoplus_{a+b=n}\left(X^{\otimes n}, \frac{1}{n !} \sum_{\sigma \in \mathfrak{S}_{n}} \sigma \sum_{i} \sigma_{i} p^{a}(1-p)^{b} \sigma_{i}^{-1}\right) .
$$

By lemma 3.5 that follows this is isomorphic to

$$
\begin{aligned}
& \bigoplus X_{a+b=n}\left(X^{\otimes n}, \frac{1}{a ! b !} \sum_{\sigma \in \mathfrak{S}_{a} \times \mathfrak{S}_{b}} \sigma p^{a}(1-p)^{b}\right) \\
= & \bigoplus_{a+b=n}\left(X^{\otimes a}, \frac{1}{a !} \sum_{\sigma \in \mathfrak{S}_{a}} \sigma p^{a}\right) \otimes\left(X^{\otimes b}, \frac{1}{b !} \sum_{\sigma \in \mathfrak{S}_{b}} \sigma(1-p)^{b}\right) \\
= & \bigoplus_{a+b=n} \lambda^{a} M \otimes \lambda^{b} N .
\end{aligned}
$$

This proves the theorem.

Lemma 3.5. There is a natural isomorphism

$$
\left(X^{\otimes n}, \frac{1}{n !} \sum_{\sigma \in \mathfrak{S}_{n}} \sigma \sum_{i} \sigma_{i} p^{a}(1-p)^{b} \sigma_{i}^{-1}\right) \stackrel{f}{\longrightarrow}\left(X^{\otimes n}, \frac{1}{a ! b !} \sum_{\sigma \in \mathfrak{S}_{a} \times \mathfrak{S}_{b}} \sigma p^{a}(1-p)^{b}\right) .
$$

Proof. Define $f \in \operatorname{End}_{\mathcal{C}} X^{\otimes n}$ to be

$$
f:=\sum_{i} p^{a} \times(1-p)^{b} \circ \sigma_{i}
$$

Recall that in a pseudoabelian category

$$
\operatorname{Hom}\left(\left(X, q_{1}\right),\left(Y, q_{2}\right)\right)=\frac{\left\{f \in \operatorname{Hom}_{\mathcal{C}}(X, Y) \mid f \circ q_{1}=q_{2} \circ f\right\}}{\left\{f \in \operatorname{Hom}_{\mathcal{C}}(X, Y) \mid f \circ q_{1}=q_{2} \circ f=0\right\}} .
$$

So we need to see that $f \circ q_{1}=q_{2} \circ f$, where $q_{1}$ and $q_{2}$ are the two projectors in the statement of the lemma,

$$
\begin{aligned}
q_{2} \circ f & =\frac{1}{a ! b !} \sum_{h \in \mathfrak{S}_{a} \times \mathfrak{S}_{b}} h p^{a}(1-p)^{b} \circ \sum_{i} p^{a}(1-p)^{b} \sigma_{i}^{-1}= \\
& =\frac{1}{a ! b !} \sum_{h \in \mathfrak{S}_{a} \times \mathfrak{S}_{b}, i} p^{a}(1-p)^{b} h \sigma_{i}^{-1}= \\
& =\frac{1}{a ! b !} p^{a}(1-p)^{b} \sum_{\sigma \in \mathfrak{S}_{n}} \sigma .
\end{aligned}
$$


On the other hand

$$
\begin{aligned}
f \circ q_{1} & =\sum_{i} p^{a}(1-p)^{b} \sigma_{i} \circ \frac{1}{n !} \sum_{\sigma \in \mathfrak{S}_{n}} \sigma \sum_{i} \sigma_{i}^{-1} p^{a}(1-p)^{b} \sigma_{i}^{-1}= \\
& =\frac{1}{n !} \sum_{\sigma \in \mathfrak{S}_{n}, i} p^{a}(1-p)^{b} \sigma_{i}^{-1} \sigma \sum_{i} \sigma_{i} p^{a}(1-p)^{b} \sigma_{i}^{-1}= \\
& =\frac{1}{n !} \frac{n !}{a ! b !} \sum_{\sigma \in \mathfrak{S}_{n}} p^{a}(1-p)^{b} \sigma^{-1} \sum_{i} \sigma_{i}^{-1} p^{a}(1-p)^{b} \sigma_{i}^{-1}= \\
& =\frac{1}{a ! b !} \sum_{h \in \mathfrak{S}_{a} \times \mathfrak{S}_{b}, i} h^{-1} p^{a}(1-p)^{b} \sigma_{i}^{-1} \sum_{i} \sigma_{i} p^{a}(1-p)^{b} \sigma_{i}^{-1}= \\
& =\frac{1}{a ! b !} \sum_{h \in \mathfrak{S}_{a} \times \mathfrak{S}_{b}, i} h^{-1} \sigma_{i}^{-1}\left(\sigma_{i} p^{a}(1-p)^{b} \sigma_{i}^{-1} \sum_{i} \sigma_{i} p^{a}(1-p)^{b} \sigma_{i}^{-1}\right)= \\
& =\frac{1}{a ! b !} \sum_{h, i} h^{-1} p^{a}(1-p)^{b} \sigma_{i}^{-1}=\frac{1}{a ! b !} p^{a}(1-p)^{b} \sum_{\sigma \in \mathfrak{S}_{n}} \sigma .
\end{aligned}
$$

This proves that $f$ defines a morphism. We show now that $f$ is an isomorphism by giving its inverse. Define $g$ to be

$$
\frac{a ! b !}{n !} \sum_{i} \sigma_{i} p^{a}(1-p)^{b} .
$$

It suffices to prove that $f \circ g=I d$ and $g \circ f=I d$. It is easy to deduce from the expression for $\operatorname{Hom}_{\mathcal{C}}\left(\left(X, q_{1}\right),\left(Y, q_{2}\right)\right)$ quoted that $f \in \operatorname{Hom}_{\mathcal{C}}(X, X)$ represents the identity of $(X, q)$ if $q \circ f=q$ or $f \circ q=q$, we shall use this in the course of the proof.

$$
\begin{aligned}
f \circ g & =\frac{a ! b !}{n !} p^{a}(1-p)^{b} \sum_{i, j} \sigma_{i} \sigma_{j}^{-1} p^{a}(1-p)^{b}= \\
& =\frac{a ! b !}{n !} p^{a}(1-p)^{b} \frac{n !}{a ! b !} i d p^{a}(1-p)^{b}=p^{a}(1-p)^{b}=I d, \\
g \circ f & =\frac{a ! b !}{n !} \sum_{i, j} \sigma_{i} p^{a}(1-p)^{b} \sigma_{j}^{-1}= \\
& =\frac{a ! b !}{n !} \sum_{i, j} \sigma_{i} p^{a}(1-p)^{b} \sigma_{j}^{-1} \circ \frac{1}{n !} \sum_{\sigma \in \mathfrak{S}_{n}} \sigma= \\
& =\frac{a ! b !}{n !} \frac{1}{n !} \sum_{i} \sigma_{i} p^{a}(1-p)^{b} \frac{n !}{a ! b !} \sum_{\sigma \in \mathfrak{S}_{n}} \sigma= \\
& =\frac{1}{n !} \sum_{i} \sigma_{i} p^{a}(1-p)^{b} \sigma_{i} \sum_{\sigma \in \mathfrak{S}_{n}} \sigma=I d .
\end{aligned}
$$

This proves the lemma

3.3. A motivic MacDonald theorem. Let $X$ be a compact polyhedron. By using the representation theory of the symmetric groups; MacDonald proved the following expression 
that relates the Betti numbers of $X$ and its symmetric powers:

$$
\sum_{n=0}^{\infty} P_{t} X^{(n)} \cdot T^{n}=\frac{(1+t T)^{b_{1}} \cdot\left(1+t^{3} T\right)^{b_{3}} \cdots}{(1-T)^{b_{0}} \cdot\left(1-t^{2} T\right)^{b_{2}} \cdots}
$$

where $b_{i}$ is the $i$-th Betti number of $X$. In this section we will generalise this result by applying Theorem 3.4 to the case in which $\mathcal{C}$ is the category of Chow motives, $\mathcal{M}_{k}$.

3.3.1. Chow motives. Let $X$ be a smooth projective variety. If $\operatorname{dim} X>1$, the symmetric power $X^{(n)}$ is a singular variety, its singularities are however very mild. In fact it is easy to extend the theory of motives to this setup. This way we obtain a functor from the category of varieties which are quotients of smooth projective varieties by finite groups to $\mathcal{M}_{k}$ we still note this functor by $h([12])$.

Proposition 3.6. The Chow motive of the symmetric power $X^{(n)}$ is naturally isomorphic to $\lambda^{n} h X$.

Proof. From the definition $\lambda^{n} h(X)=h\left(X^{n}\right)^{\mathfrak{S}_{n}}$, as a consequence of the proof of proposition 1.2 in [12] this is isomorphic to $h\left(X^{n} / \mathfrak{S}_{n}\right) \in \mathrm{Ob} \mathcal{M}_{k}^{+}$.

MacDonald's formula ([29]) relates the Betti numbers of $X^{(n)}$ to those of $X$. The motivic counterpart of the Betti numbers are the factors in the conjectural weight decomposition $h X=$ $h^{0} X \oplus \cdots \oplus h^{2 \operatorname{dim} X} X$ described, for instance, in [36], 6.2. Thus we can only expect to prove an analogous to MacDonald's formula if we assume such a decomposition exists. This explains the hypothesis in the following proposition. A similar result for the case $X$ is a smooth projective curve has been obtained by S̆hermenev ([37], Proposition 4).

Proposition 3.7. Let $X$ be a smooth projective variety and $n$ a positive integer. Assume $h X$ has a decomposition

$$
h X=h^{0} X \oplus h^{1} X \oplus \cdots \oplus h^{2 \operatorname{dim} X} X
$$

as described above. Then the motive of the n-fold symmetric power of $X$ is isomorphic to

$$
\bigoplus_{n_{0}+\cdots+n_{2 \operatorname{dim} X}=n} \lambda^{n_{0}} h^{0} X \otimes \cdots \otimes \lambda^{n_{2} \operatorname{dim} X} h^{2 \operatorname{dim} X} X
$$

Moreover $X^{(n)}$ also has a decomposition of the above type with

$$
h^{i} X^{(n)} \simeq \bigoplus_{\begin{array}{c}
n_{0}+\cdots+n_{2 d}=n \\
n_{1}+2 n_{2}+\cdots+2 d n_{2 d}=i
\end{array}} \lambda^{n_{0}} h^{0} X \otimes \cdots \otimes \lambda^{n_{2 d}} h^{2 d} X .
$$

Proof. By Proposition 3.6 $h X^{(n)}=\lambda^{n} h X$. Repeated application of theorem 3.4 to the decomposition of $h X$ in the hypothesis yield the isomorphism (7).

To prove the second assertion call $M_{i}$ the motive on the right hand side of (8) and note that $h X^{(n)}=\oplus M_{i}$, it is easy to see that $H_{e t}^{i}\left(h X^{(n)}, \mathbb{Q}_{\ell}\right) \simeq H_{e t}^{*}\left(M_{i}, \mathbb{Q}_{\ell}\right)$, this implies that the cohomology classes of the projectors defining the direct summands $M_{i}$ in $h X^{(n)}$ are the Künneth components of the diagonal class.

The previous proposition applied to the category of motives modulo an homological equivalence shows that if $X$ verifies standard conjecture $C([20])$ so does $X^{(n)}$. 
3.3.2. Cohomology. Let $H^{*}: \mathcal{M}_{k} \longrightarrow \mathcal{C}$ be a realisation functor. We have the following generalisation of MacDonald's formula.

Proposition 3.8. Let $X$ be a smooth projective variety of dimension d, we have the following isomorphism

$$
H^{i}\left(X^{(n)}\right) \simeq \bigoplus_{\begin{array}{c}
n_{0}+\cdots+n_{2 d}=n \\
n_{1}+2 n_{2}+\cdots+2 d n_{2 d}=i
\end{array}}\left(\bigotimes_{k \text { even }} S^{n_{k}} H^{k}(X) \otimes \bigotimes_{k \text { odd }} \wedge^{n_{k}} H^{k}(X)\right)
$$

Proof. By functoriality, $H^{*}\left(X^{(n)}\right)=H^{*}\left(\lambda^{n} h(X)\right)$. But this is not isomorphic to $\lambda^{n} H^{*}(X)$ because the functor $H^{*}$ is not a tensor functor $([13])$. This is so because if

$$
c_{M N}: H^{*} N \otimes H^{*} N \stackrel{\simeq}{\longrightarrow} H^{*}(M \otimes N)
$$

is the Künneth isomorphism. Then the diagram

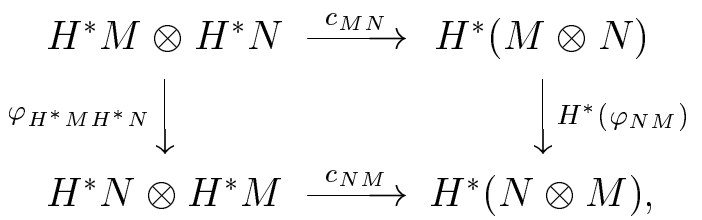

where vertical arrows are the commutativity constraints in each category, does not, in general, commute.

To remedy this we change the commutation constraints in $\mathcal{C}$. Let $\mathcal{C}^{\bullet}$ be the category $\mathcal{C}$ with the commutativity constraints given by

$$
(-1)^{i j} \varphi_{M N}: M \otimes N \longrightarrow N \otimes M
$$

on objects $M, N$ of pure weights $i$ and $j$ respectively. Then the functor $H^{*}: \mathcal{M}_{k} \longrightarrow \mathcal{C}^{\bullet}$ is a tensor functor and we thus have $H^{*} \lambda^{n} h X \simeq \lambda^{n} H^{*} X$ where $\lambda^{n}$ is performed in the category $\mathcal{C}^{\bullet}$. But by iterated application of theorem 3.4

$$
\lambda^{n} H^{*} X \simeq \bigoplus_{n_{0}+n_{1}+\cdots+n_{2 d}=n} \lambda^{n_{0}} H^{0} X \otimes \lambda^{n_{1}} H^{1} X \otimes \cdots \otimes \lambda^{n_{2 d}} H^{2 d} X .
$$

If $M$ is an object of $\mathcal{C}^{\bullet}$ of even weight the commutation constraints $\mathfrak{S}_{n} \longrightarrow \operatorname{Aut}\left(M^{\otimes n}\right)$ in $\mathcal{C}^{\bullet}$ coincide with the usual ones in $\mathcal{C}$, this implies that for even $j, \lambda^{n}\left(H^{j}(X)\right)$ is the ordinary symmetric power $S^{n}\left(H^{j}(X)\right)$.

On the other hand if $M$ is of odd weight the commutation constraints $\mathfrak{S}_{n} \longrightarrow$ Aut $\left(M^{\otimes n}\right)$ in $\mathcal{C}^{\bullet}$ are the ordinary ones multiplied by the signature morphism $\mathfrak{S}_{n} \longrightarrow\{ \pm 1\}$. This implies that for odd $j \lambda^{n}\left(H^{j}(X)\right)$ is the wedge $\wedge^{n}\left(H^{j}(X)\right)$.

This shows that

$$
H^{*} X^{(n)} \simeq \bigoplus_{n_{0}+n_{1}+\cdots+n_{2 d}=n} S^{n_{0}} H^{0} X \otimes \wedge^{n_{1}} H^{1} X \otimes \cdots \otimes S^{n_{2 d}} H^{2 d} X .
$$

The statement of the proposition results from taking the part of degree $i$.

In the case that $\mathcal{C}$ is the category of pure Hodge structures over $\mathbb{C}, \mathbf{P H} \mathbf{S}_{\mathbb{C}}$, and $H^{*}$ is the cohomology functor $\mathcal{V}_{k} \longrightarrow \mathbf{P H S}_{\mathbb{C}}$ the previous proposition yields an isomorphism

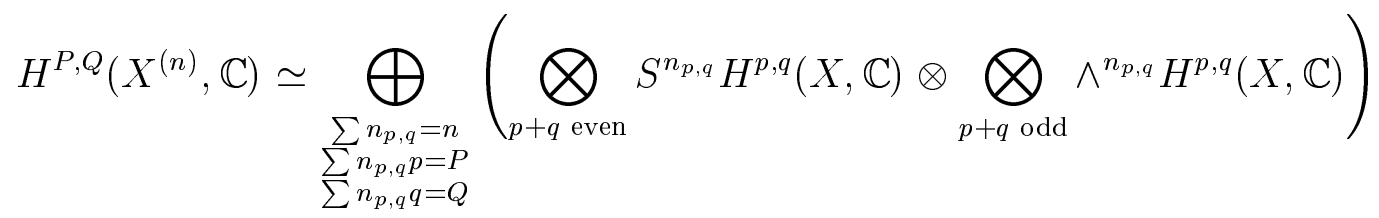


for $P, Q \in \mathbb{N}$. Taking the class of this in $K_{0}\left(\mathbf{P H S}_{\mathbb{C}}\right)=\mathbb{Z}\left[x, y, x^{-1}, y^{-1}\right]$ and using the generating functions

$$
\begin{aligned}
& \sum \operatorname{dim} \wedge^{i} \mathbb{C}^{n} \cdot T^{i}=(1+T)^{n} \\
& \sum \operatorname{dim} S^{i} \mathbb{C}^{n} \cdot T^{i}=\frac{1}{(1-T)^{n}}
\end{aligned}
$$

proves the following generalisation of MacDonald's formula obtained by J. Burillo by representation theoretic methods.

Corollary 3.9. ([11]) If $h_{p q}(X)=\operatorname{dim} H^{p, q}(X, \mathbb{C})$ are the Hodge numbers of $X$ then the Poincaré-Hodge polynomial of $X^{(n)}$ is

$$
P_{x y}\left(X^{(n)}\right)=\operatorname{Coef}_{T^{n}} \prod_{p, q}\left(1-(-1)^{p+q} x^{p} y^{q} T\right)^{(-1)^{p+q+1} h_{p q}(X)} .
$$

\section{The MOTIVE OF MODUli SPACES OF VECTOR BUNDLES}

4.1. On work by Bifet, Letizia and Ghione. We briefly recall some of the main results in $[5]$.

Let $C$ be a smooth projective curve over a field $k$. Recall that a matrix divisor of rank $n$ over $C$ is a rank $n$ vector bundle $\mathcal{E}$ together with an inclusion of $\mathcal{O}_{C}$-modules $\iota: \mathcal{E} \hookrightarrow \mathcal{K}_{C}^{n}$, here $\mathcal{K}_{C}$ notes the constant sheaf of rational functions on $C$. Given a matrix divisor $\iota: \mathcal{E} \hookrightarrow \mathcal{K}_{C}^{n}$ we can always find an effective divisor $D$ such that $\iota$ factors via $\mathcal{O}_{C}(D)^{n} \subset \mathcal{K}_{C}^{n}$.

Fix an integer $d$. Given an effective divisor $D$ over $C$, the set of rank $n$ matrix divisors of degree $d$ that lie in $\mathcal{O}_{C}(D)^{n}$ is the set of $k$-points of the Quot scheme $Q_{n, d}(D)=Q u o t_{\mathcal{O}_{C}^{n}(D)|C| k}^{n \operatorname{deg} D-d}$, this is a smooth projective irreducible variety. If $D<D^{\prime}$ we have a natural closed immersion $Q_{n, d}(D) \subset Q_{n, d}\left(D^{\prime}\right)$. This defines an ind-variety $Q_{n, d}=\left\{Q_{n, d}(D)\right\}_{D}$ which is called the indvariety of matrix divisors of rank $n$ and degree $d$.

The universal bundle on these Quot schemes gives rise to a Shatz stratification according to the Harder-Narasimhan type of the underlying vector bundle.

$$
Q_{n, d}=\bigcup_{\mathbf{n}, \mathbf{d}} Q_{\mathbf{n}, \mathbf{d}}^{s s}
$$

where $(\mathbf{n}, \mathbf{d})=\left(\left(n_{1}, d_{1}\right), \ldots,\left(n_{l}, d_{l}\right)\right)$ is the Shatz polygon.

Theorem 4.1. $\quad$ 1. ([5], 4.2) The cohomology of $Q_{n, d}$ stabilizes. Its Poincaré series converges to

$$
\frac{(1+t)^{2 g}}{1-t^{2 n}} \prod_{i=1}^{n-1} \frac{\left(1+t^{2 i-1}\right)^{2 g}}{\left(1-t^{2 i}\right)^{2}} .
$$

2. ([5], 7.1) For each Shatz polygon $(\mathbf{n}, \mathbf{d})$ the cohomology of the strata $Q_{\mathbf{n}, \mathbf{d}}^{\text {ss }}$ stabilizes. There is a natural morphism $\prod_{i} Q_{n_{i}, d_{i}}^{s s} \longrightarrow Q_{\mathbf{n}, \mathbf{d}}^{s s}$ that induces an isomorphism on cohomology.

3. ([5], 8.1) If $n$ and $d$ are coprime $P_{t} Q_{n, d}^{s s}=\frac{1}{1-t^{2}} P_{t} N_{C}(n, d)$.

The stratification (11) is proven to be perfect in the sense that all the Gysin exact sequences split in short exact sequences (see [5] $\S 10$ for details). The main outcome of this is the following formula,

$$
P_{t} Q_{n, d}=\sum_{\substack{\mathbf{n}=\left(n_{1}, \ldots, n_{l}\right) \\ \mathbf{d}=\left(d_{1}, \ldots, d_{l}\right)}} P_{t} Q_{n_{1}, d_{1}}^{s s} \cdots P_{t} Q_{n_{l}, d_{l}}^{s s} t^{2 c(\mathbf{n}, \mathbf{d})}
$$


where $(\mathbf{n}, \mathbf{d})$ runs over the Shatz polygons and

$$
c(\mathbf{n}, \mathbf{d})=\sum_{i>j}\left(n_{i} d_{j}-n_{j} d_{i}+n_{i} n_{j}(g-1)\right) .
$$

This expression combined with theorem 4.1 .2 can be used to compute $P_{t} Q_{n, d}^{s s}$ inductively.

4.2. Chow motive of varieties of matrix divisors. We shall give a motivic version of the results of E. Bifet ([4]) concerning the scheme $Q u o t_{\mathcal{O}_{C}^{n}|C| k}^{d}$. The key to the results in [4] is the use of a torus action and the results of Bialynicki-Birula, using theorem 2.4 we can now prove:

Theorem 4.2. The Chow motive of Quot $_{\mathcal{O}_{C}^{n}|C| k}^{d}$ is isomorphic to the Chow motive of the d-fold symmetric power of $C \times \mathbb{P}^{n-1}$,

$$
h Q u o t_{\mathcal{O}_{C}^{n}|C| k}^{d} \simeq \lambda^{d} h\left(C \times \mathbb{P}^{n-1}\right) .
$$

The same holds for the Chow groups and algebraic K-theory with rational coefficients.

Proof. Clearly automorphism group of $\mathcal{O}_{C}^{n}, G L_{n}(k)$, acts on the Quot scheme. By [4] we know that the action of the standard maximal torus of $G L_{n}(k)$ on $Q u o t$ has fixed points of the form $\mathcal{O}_{C}\left(-D_{1}\right) \oplus \cdots \mathcal{O}_{C}\left(-D_{n}\right) \hookrightarrow \mathcal{O}_{C}^{n}$ where $D_{1}, \ldots, D_{n}$ are effective divisors. This fixed point set remains the same if we restrict the action to a sufficiently general one parameter subgroup. In sum, $\mathbb{G}_{m}$ acts on this Quot scheme with fixed point set

$$
\left(\text { Quot }_{\mathcal{O}_{C}^{n}|C| k}^{d}\right)^{\mathbb{G}_{m}}=\bigcup_{d_{1}+\cdots+d_{n}=d} C^{\left(d_{1}\right)} \times \cdots \times C^{\left(d_{n}\right)}
$$

Moreover, the codimension of the stratum corresponding to $\left(d_{1}, \ldots, d_{n}\right)$ is $\sum(i-1) d_{i}$.

Now our localisation theorem 2.4.(4) together with theorem 3.4 yields

$$
\begin{aligned}
h Q u o t_{\mathcal{O}_{C}^{n}|C| k}^{d} & \simeq \sum_{d_{1}+\cdots+d_{n}=d} h\left(C^{\left(d_{1}\right)} \times \cdots \times C^{\left(d_{n}\right)}\right)\left(\sum(1-i) d_{i}\right) \\
& \simeq \sum_{d_{1}+\cdots+d_{n}=d} \lambda^{d_{1}}(h C(1-1)) \otimes \cdots \otimes \lambda^{d_{n}}(h C(1-n)) \\
& \simeq \lambda^{d}(h C \oplus h C(-1) \oplus \cdots \oplus h C(1-n)) \\
& \simeq h\left(\left(C \times \mathbb{P}^{n-1}\right)^{(d)}\right) .
\end{aligned}
$$

The statements for Chow groups and $K$-theory are proved in the same fashion.

It should be pointed out that $\left(C \times \mathbb{P}^{n-1}\right)^{(d)}$ is a singular variety and the previous isomorphism between motives cannot come from an isomorphism of varieties.

Definition 4.3. Let $C$ be a smooth projective curve over $k$ of genus $g$ define $\left(\mathbb{1}+\mathbb{L}^{n}\right)^{h^{1}(C)}$ to be $\oplus_{i=0}^{2 g} \lambda^{i} h^{1} C \otimes \mathbb{L}^{n i}$.

Proposition 4.4. $\quad$ 1. The motivic Poincaré polynomial of $Q_{n, d}$ stabilises. It converges to

$$
\frac{(\mathbb{1}+\mathbb{1})^{h^{1} C}}{\left(\mathbb{1}-\mathbb{L}^{n}\right)} \prod_{i=1}^{n-1} \frac{\left(\mathbb{1}+\mathbb{L}^{i}\right)^{h^{1} C}}{\left(\mathbb{1}-\mathbb{L}^{i}\right)^{2}} .
$$

2. For each Shatz polygon $(\mathbf{n}, \mathbf{d})$ the motivic Poincaré polynomial of the strata $Q_{\mathbf{n}, \mathbf{d}}$ stabilises and $\chi Q_{\mathbf{n}, \mathbf{d}}^{s s}=\prod_{i} \chi Q_{n_{i}, d_{i}}^{s s}$.

3. If $n$ and $d$ are coprime $\chi Q_{n, d}^{s s}=\frac{1}{1-\mathbb{L}} \chi N_{C}(n, d)$. 
Proof. 1. First note that tensoring by $\mathcal{O}_{C}(-D)$ defines an isomorphism

$$
Q u o t_{\mathcal{O}_{C}^{n}(D) / C / k}^{d} \simeq Q u o t_{\mathcal{O}_{C}^{n} / C / k}^{d}
$$

In Theorem 4.2 we have proved that

$$
h Q u o t_{\mathcal{O}_{C}^{n} / C / k}^{d} \simeq \lambda^{d}\left(h\left(\mathbb{P}^{n-1} \times C\right)\right),
$$

By splitting off a trivial motive as in [36] 1.11, $h\left(\mathbb{P}^{n-1} \times C\right)=\mathbb{1} \oplus h^{+}\left(\mathbb{P}^{n-1} \times C\right)$, and using Theorem 3.4 we see that $h Q u \operatorname{uot}_{\mathcal{O}_{C}^{n}|C| k}^{d}=\otimes_{i \leq d} \lambda^{i}\left(h^{+}\left(\mathbb{P}^{n-1} \times C\right)\right)$. From this we see that

$$
\begin{aligned}
& \chi Q u o t_{\mathcal{O}_{C}^{n} / C / k}^{d+1}-\chi Q u o t_{\mathcal{O}_{C}^{n} / C / k}^{d}=\lambda^{d+1}\left(\chi\left(\mathbb{P}^{n-1} \times C\right)-\mathbb{1}\right) \\
& \in \mathbb{L}^{d+1} \cdot K_{0} \mathcal{M}_{k}^{+} \text {. }
\end{aligned}
$$

This implies that $\left(\chi Q_{n, d}(D)\right)_{D}$ converges. Moreover, using again the additivity of the $\lambda$-structure (Theorem 3.4) we obtain

$$
\begin{aligned}
\sum_{i=0}^{\infty} \lambda^{i}\left(h^{+}\left(\mathbb{P}^{n-1} \times C\right)\right) \simeq & \sum_{i=0}^{\infty} \lambda^{i}\left(h \mathbb{P}_{k}^{n-1}(-1) \oplus h \mathbb{P}_{k}^{n-2}(-1) \oplus\left(h \mathbb{P}_{k}^{n-1} \otimes h^{1} C\right)\right) \\
\simeq & \left(\oplus_{i=0}^{\infty} \lambda^{i} \mathbb{L}\right) \cdots\left(\oplus_{i=0}^{\infty} \lambda^{i} \mathbb{L}^{n}\right) \cdot\left(\oplus_{i=0}^{\infty} \lambda^{i} \mathbb{L}\right) \cdots\left(\oplus_{i=0}^{\infty} \lambda^{i} \mathbb{L}^{n-1}\right) \cdot \\
& \cdot\left(\oplus_{i=0}^{\infty} \lambda^{i} h^{1} C\right) \cdots\left(\oplus_{i=0}^{\infty} \lambda^{i}\left(h^{1} C(-n+1)\right)\right) \\
\simeq & \frac{(1+11)^{h^{1} C}}{\left(\mathbb{1}-\mathbb{L}^{n}\right)} \prod_{i=1}^{n-1} \frac{\left(1+\mathbb{L}^{i}\right)^{h^{1} C}}{\left(1-\mathbb{L}^{i}\right)^{2}} .
\end{aligned}
$$

2. This follows from the proof of theorem 7.1 in [5].

3. Let $\mathcal{P}$ be a Poincaré bundle on $N_{C}(n, d) \times C$ and let $p_{1}$ and $p_{2}$ be the projections of $N_{C}(n, d) \times C$ on its factors. According to [5] there is a morphism

$$
Q_{n, d}^{s s}(D) \stackrel{i_{D}}{\longrightarrow} \mathbb{P}(D):=\mathbb{P} p_{1 *}\left(\mathcal{P}^{\vee} \otimes p_{2}^{*} \mathcal{O}_{C}(D)^{n}\right)
$$

which is an open immersion with complementary closed subset of codimension $\geq \operatorname{deg} D$. Then corollary 4.7 implies that $\left(\chi Q_{n, d}^{s s}(D)-\chi \mathbb{P}(D)\right)_{D}$ converges to zero. But $\mathbb{P}(D)$ is a projective bundle of rank $n^{2} \operatorname{deg} D-n d$ over $N_{C}(n, d)$ so $(\chi \mathbb{P}(D))_{D}$ converges to $\frac{1}{1-\mathbb{L}} \chi N_{C}(n, d)$.

4.3. The motive of $N_{C}(n, d)$. Let $A \subset \mathrm{Ob}\left(\mathcal{M}_{k}^{+}\right)$be a collection of effective motives. We define the pseudoabelian tensor category of $\mathcal{M}_{k}^{+}$generated by $A$ to be the full subcategory of $\mathcal{M}_{k}^{+}$whose objects are direct summands of sums of tensor products of motives in $A$.

The following result shows can the theory of [21] can be applied to classical motives.

Theorem 4.5. Let $C$ be a smooth projective curve over a field of characteristic zero and let $n>1$ and d be coprime integers. Then the Chow motive $h N_{C}(n, d)$ lies in the full pseudoabelian tensor category of $\mathcal{M}_{k}^{+}$generated by $h^{1}(C)$ and $\mathbb{L}$.

The proof of the theorem uses the following result.

Lemma 4.6. Let $Y$ be a closed subvariety of codimension d of a smooth quasiprojective irreducible variety $X$. Then

1. If $Y$ is smooth then there is an isomorphism in $H o C^{b} \mathcal{M}_{k}^{+}, h(X-Y) \simeq \mathbf{s}(h Y(-d) \longrightarrow h X)$.

2. There exists a complex of effective motives $C^{\bullet}$ concentrated in negative degree and an isomorphism in $\mathrm{Ho}^{b} \mathcal{M}_{k}^{+}, h(X-Y) \simeq \mathbf{s}\left(C^{\bullet}(-d) \longrightarrow h X\right)$. 
By taking classes in $K_{0} \mathcal{M}_{k}^{+}$we obtain the following corollary.

Corollary 4.7. Let $Y$ be a closed subvariety of codimension d of a smooth quasiprojective irreducible variety $X$. Then

1. If $Y$ is smooth then $\chi(X)-\chi(X-Y)=\chi(Y)(-d)$.

2. In any case $\chi(X)-\chi(X-Y)$ lies in the ideal of $K_{0} \mathcal{M}_{k}^{+}$generated by $\mathbb{L}^{d}$.

Proof of lemma. 1. Recall from Proposition 6.4.2 in [12] that for each smooth quasiprojective variety $U$, we have an isomorphism $h(U) \simeq h_{c}(U)^{\vee}(-\operatorname{dim} U)$. Therefore

$$
\begin{aligned}
h(X-Y) & \simeq h_{c}(X-Y)^{\vee}(-\operatorname{dim} X) \simeq \mathrm{s}\left(h_{c}(X) \longrightarrow h_{c}(Y)\right)^{\vee}(-\operatorname{dim} X) \\
& \simeq \mathrm{s}\left(h_{c}(Y)^{\vee} \longrightarrow h_{c}(X)^{\vee}\right)(-\operatorname{dim} X) \simeq \mathrm{s}(h(Y)(-d) \longrightarrow h(X)) .
\end{aligned}
$$

2. The proof is by induction on $\operatorname{dim} Y$. The statement is obvious for $\operatorname{dim} Y=-1$ (i.e. $Y=\emptyset)$ so we assume $\operatorname{dim} Y \geq 0$. Let $Y^{\operatorname{sing}} \subset Y$ be the singular locus of $Y$, we have $\operatorname{dim} Y^{\operatorname{sing}}<\operatorname{dim} Y$. By the smooth case we have

$$
h(X-Y) \simeq \mathbf{s}\left(h\left(Y-Y^{\text {sing }}\right)(-d) \longrightarrow h\left(X-Y^{\text {sing }}\right)\right)
$$

and from the induction hypothesis we have a complex of effective motives $C_{0}^{\bullet}$ concentrated in negative degree and an isomorphism

$$
h\left(X-Y^{\text {sing }}\right) \simeq \mathbf{s}\left(C_{0}^{\bullet}(-d-1) \longrightarrow h(X)\right) .
$$

Putting this together we get

$$
h(X-Y) \simeq \mathbf{s}\left(h\left(Y-Y^{\text {sing }}\right)(-d) \longrightarrow \mathbf{s}\left(C_{0}^{\bullet}(-d-1) \longrightarrow h(X)\right)\right)
$$

Next lemma provides us with an isomorphism

$$
\begin{aligned}
\mathrm{s}\left(h ( Y - Y ^ { \text { sing } } ) ( - d ) \longrightarrow \mathrm { s } \left(C_{0}^{\bullet}(\right.\right. & -d-1) \longrightarrow h(X))) \simeq \\
& \simeq \mathrm{s}\left(\mathrm{s}\left(h\left(Y-Y^{\text {sing }}\right)(-d)[-1] \longrightarrow C_{0}^{\bullet}(-d-1)\right) \longrightarrow h(X)\right)
\end{aligned}
$$

The proof of the lemma follows if we define $C^{\bullet}$ to be the simple of the morphism $h(Y-$ $\left.Y^{\text {sing }}\right)[-1] \longrightarrow C_{0}^{\bullet}(-1)$ by noting that the graded object underlying the complex $C^{\bullet}$ is is the same as that of $C_{0}^{\bullet}(-1) \oplus h\left(Y-Y^{\text {sing }}\right)$ which is concentrated in negative degrees.

Lemma 4.8. Let $A, B$ and $C$ be complexes of chains in an additive category $\mathcal{C}$ and let $f$ : $B \longrightarrow C, G: A \longrightarrow \mathrm{s}(B \stackrel{f}{\longrightarrow} C)$ be morphisms of complexes. There exist morphisms of complexes $g$ and $F$ and an isomorphism of complexes

$$
\mathbf{s}(A \stackrel{G}{\rightarrow} \mathbf{s}(B \stackrel{f}{\rightarrow} C)) \simeq \mathbf{s}(\mathbf{s}(A[-1] \stackrel{g}{\rightarrow} B) \stackrel{F}{\rightarrow} C)
$$

Proof. To give a morphism $G: A \longrightarrow \mathbf{s}(B \stackrel{f}{\longrightarrow} C)$ is equivalent to giving a morphism of graded objects in $\mathcal{C},\left(\begin{array}{l}g^{\prime} \\ h\end{array}\right): A \longrightarrow B[1] \oplus C$ such that

$$
\left(\begin{array}{l}
g^{\prime} \\
h
\end{array}\right) d_{A}=\left(\begin{array}{cc}
d_{B[1]} & 0 \\
f & d_{C}
\end{array}\right)\left(\begin{array}{l}
g^{\prime} \\
h
\end{array}\right)
$$

that is

$$
g^{\prime} \circ d_{A}=d_{B[1]} \circ g^{\prime} \quad \text { y } \quad h \circ d_{A}-d_{C} \circ h=f \circ g^{\prime}
$$

(note incidentally that this is equivalent to $g^{\prime}$ defining a morphism of complexes and $h$ an homotopy $f \circ g^{\prime} \sim 0$ ). 
The simple of $G$ is the complex with underlying graded object $A[1] \oplus B[1] \oplus C$ and differentials given by

$$
\left(\begin{array}{ccc}
d_{A[1]} & 0 & 0 \\
g^{\prime} & d_{B[1]} & 0 \\
h & f & d_{C}
\end{array}\right) .
$$

Note that the simple of $g=-g^{\prime}=g[-1]: A[-1] \longrightarrow B$ is the complex with $A \oplus B$ as underlying graded object and differential $\left(\begin{array}{cc}d_{A} & 0 \\ -g^{\prime} & d_{B}\end{array}\right)$.

Define $F: \mathbf{s}(A[-1] \stackrel{g}{\longrightarrow} B) \longrightarrow C$ over the graded object by $(h, f): A \oplus B \longrightarrow C$, this is a morphism of complexes for

$$
\begin{aligned}
(h, f)\left(\begin{array}{cc}
d_{A} & 0 \\
g & d_{B}
\end{array}\right) & =\left(h \circ d_{A}+f \circ g, f \circ d_{B}\right) \\
d_{C}(h, f) & =\left(d_{C} \circ h, d_{C} \circ f\right)
\end{aligned}
$$

and from the definition of $f$ and (13) these expressions coincide.

As above the simple of $F$ is $A[1] \oplus B[1] \oplus C$ with differential

$$
\left(\begin{array}{ccc}
d_{A[1]} & 0 & 0 \\
g^{\prime} & d_{B[1]} & 0 \\
h & f & d_{C}
\end{array}\right)
$$

this coincides with the simple of $G$ and the lemma is proved.

Proof of Theorem 4.5. Recall from [5] $\S 8$ that for every effective divisor $D$ on the curve $C$ we have a projective bundle $\mathbb{P}(D)$ over $N_{C}(n, d)$ associated to a vector bundle, call $r$ its relative dimension. There is an open immersion $i_{D}: Q_{n, d}^{s s}(D) \hookrightarrow \mathbb{P}(D)$ with complementary closed subset of codimension $c(D) \geq \operatorname{deg}(D)$. From the previous lemma we obtain an isomorphism

$$
h\left(Q_{n, d}^{s s}(D)\right) \simeq \mathbf{s}\left(C_{1}^{\bullet}(-c(D)) \longrightarrow h(\mathbb{P}(D))\right)
$$

where $C_{1} \bullet$ is a complex of effective motives concentrated in negative degree.

If $\operatorname{deg}(D)$ is big enough then $Q_{n, d}^{s s}(D)$ is a non empty open set of the smooth irreducible projective variety $Q_{n, d}(D)$. By the lemma there is a complex of effective motives $C_{2}^{\bullet}$ and an isomorphism

$$
h\left(Q_{n, d}^{s s}(D)\right) \simeq \mathbf{s}\left(C_{2}^{\bullet}(-1) \longrightarrow h\left(Q_{n, d}(D)\right)\right)
$$

Given that $h Q_{n, d}^{s s}(D)$ is defined up to homotopy equivalence we have morphisms of complexes

$$
\left[C_{1}^{\bullet}(-c(D)) \longrightarrow h(\mathbb{P}(D))\right] \stackrel{f^{\bullet}}{\stackrel{g^{\bullet}}{\leftrightarrows}}\left[C_{2}^{\bullet}(-1) \longrightarrow h\left(Q_{n, d}(D)\right)\right]
$$

with $f^{\bullet} \circ g^{\bullet} \sim I d$ and $g^{\bullet} \circ f^{\bullet} \sim I d$.

Next recall that the natural projection $\pi: \mathbb{P}(D) \longrightarrow N_{C}(n, d)$ provides with a monomorphism of motives $\pi^{*}: h N_{C}(n, d) \longrightarrow h \mathbb{P}(D)$ with a natural retraction given by $R=\pi_{*} \circ \overline{c_{1} \mathcal{O}_{\mathbb{P}(D)}(1)^{r}}$ : $h \mathbb{P}(D) \longrightarrow h N_{C}(n, d)$. Clearly $\pi^{*}$ defines a morphism of complexes

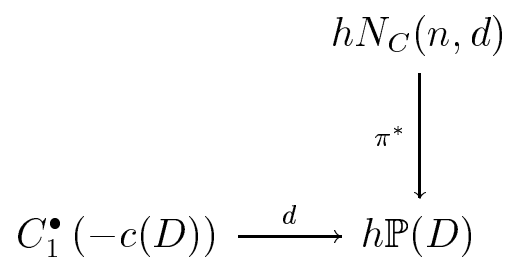


The key point now is that if $\operatorname{deg}(D)$ is big enough then

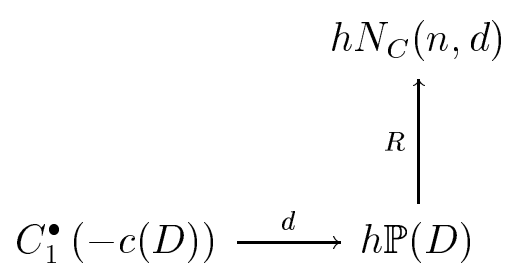

defines a morphism of complexes. This is so because if $C_{1}^{-1}=(X, p)$

$$
\begin{aligned}
R \circ d \in & \operatorname{Hom}_{\mathcal{M}_{k}^{+}}\left(C_{1}^{-1}(-c(D)), h N_{C}(n, d)\right) \subset \operatorname{Hom}_{\mathcal{M}_{k}^{+}}\left(h X(-c(D)), h N_{C}(n, d)\right) \simeq \\
& \simeq C H^{c(D)+\operatorname{dim} X}\left(X \times N_{C}(n, d)\right)=0 .
\end{aligned}
$$

This last group being zero for dimensional reasons. Therefore (15) is a monomorphism. Composing this monomorphism with the homotopy equivalence (14) gives another monomorphism with retraction given by $R \circ g$.

$$
\begin{aligned}
& h N_{C}(n, d) \\
& f^{0} \circ \pi^{*} \\
& C_{2}^{\bullet}(-1) \stackrel{d}{\longrightarrow} h Q_{n, d}(D)
\end{aligned}
$$

Therefore $R \circ g \circ f^{0} \circ \pi^{*} \sim I d_{h N_{C}(n, d)}$. But as $h N_{C}(n, d)$ is concentrated in degree zero we have $R \circ g \circ f^{0} \circ \pi^{*}=I d_{h N_{C}(n, d)}$, this implies that $f \circ \pi^{*}: h N_{C}(n, d) \longrightarrow h Q_{n, d}(D)$ is a monomorphism. By Theorem 4.2 the motive of this lies in the tensor category generated by $h^{1}(C)$ and $\mathbb{L}$. The result follows.

4.4. The motivic Poincaré polynomial of the moduli space. The following proposition is a motivic version of the classical recursion for the Betti numbers of the moduli space (c.f. [22], [23], [14] and [1]). The reader will note that no perfection arguments are needed in this context.

Proposition 4.9. We have the following recursion formula

$$
\chi Q_{n, d}=\sum_{\mathbf{n}, \mathbf{d}} \chi Q_{\mathbf{n}, \mathbf{d}}^{s s}\left(-\sum_{i<j}\left(n_{i} d_{j}-n_{j} d_{i}+n_{i} n_{j}(g-1)\right)\right),
$$

where the $(\mathbf{n}, \mathbf{d})=\left(\left(n_{1}, d_{1}\right), \cdots,\left(n_{l}, d_{l}\right)\right)$ run over the Shatz polygons.

Proof. Let $D$ be an effective divisor. Consider the Shatz stratification $Q_{n, d}(D)=\cup Q_{\mathbf{n}, \mathbf{d}}^{s s}(D)$. Call $F(D)$ the union of the strata, $Q_{\mathbf{n}, \mathbf{d}}^{s s}$, that are not smooth or not of codimension $c(\mathbf{n}, \mathbf{d})$. By Proposition 5.2.(3) in [5] $F(D)$ is a closed subvariety of codimension greater than $\operatorname{deg} D-c$ (where $c$ is a constant independent of $D$ ) so by corollary 4.7

$$
\chi Q_{n, d}(D)-\chi\left(Q_{n, d}(D)-F(D)\right) \in \mathbb{1}(-\operatorname{deg} D+c) \cdot K_{0} \mathcal{M}_{k}^{+} .
$$

If $Q_{\mathbf{n}_{1}, \mathbf{d}_{1}}^{s s}(D), \ldots, Q_{\mathbf{n}_{k}, \mathbf{d}_{k}}^{s s}(D)$ are the smooth strata of the right codimension then by corollary 4.7 we have

$$
\chi\left(Q_{n, d}(D)-F(D)\right)=\sum_{j=1}^{k} \chi Q_{\mathbf{n}_{j}, \mathbf{d}_{j}}^{s s}(D)\left(-c\left(\mathbf{n}_{j}, \mathbf{d}_{j}\right)\right) .
$$

The proposition now follows by taking the limit as $\operatorname{deg} D \rightarrow \infty$. 
We now proceed to invert this motivic recursion by using the work of Laumon and Rapoport ([28]). As in [28] if $x$ is a real number we note by $\langle x\rangle$ its decimal part, that is, its representative modulo $\mathbb{Z}$ in $[0,1)$.

To apply the inversion procedure of Laumon and Rapoport we first notice that the integers $\left\langle\delta_{P}^{Q}, \alpha^{\vee}\right\rangle$ and $n_{P}$ introduced in [28] (section 2) are in fact in $2 \mathbb{Z}$ in the case of vector bundles. This observation allows one to state and prove a version of theorem 2.4 in [28] substituting $\mathbb{Z}[[t]]$ by $\mathcal{K}$ and $t^{m\left(P, \nu_{P}^{\prime}\right)}$ by $\mathbb{1}\left(\frac{m\left(P, \nu_{P}^{\prime}\right)}{2}\right)$.

We now introduce some notation to state the inversion theorem in the special case of vector bundles. The standard parabolic subgroups of $G L_{n} k$ can be identified with the set $\mathcal{P}_{n}$ of partitions of $n$ in sum of positive integers. There is a natural partial order in $\mathcal{P}_{n}$, $\mathbf{n}^{\prime}=\left(n_{1}, \cdots, n_{s}\right) \subset \mathbf{n}=\left(n_{1}, \cdots n_{l}\right)$ whenever there exist integers $0=k_{0}<\cdots<k_{l}$ with $n_{k_{i}+1}^{\prime}+\cdots+n_{k_{i+1}}^{\prime}=n_{i+1}$ for each $i \in\{0, \cdots, l-1\}$. Define the function $\ell: \mathcal{P}_{n} \rightarrow \mathbb{N}$ to be the length of a partition.

Given an integer $d$ let $\mathcal{P}_{n, d}$ be the set $\left\{(\mathbf{n}, \mathbf{d}) \mid \mathbf{n} \in \mathcal{P}_{n}, \mathbf{d} \in \mathbb{Z}^{\ell(\mathbf{n})}, \sum d_{i}=d\right\}$. We set $\left(\mathbf{n}^{\prime}, \mathbf{d}^{\prime}\right) \subset(\mathbf{n}, \mathbf{d})$ whenever $\mathbf{n}^{\prime} \subset \mathbf{n}$ and in the previous notation $d_{k_{i}+1}^{\prime}+\cdots+d_{k_{i}+1}^{\prime}=d_{i}$ for $i \in\{0, \cdots, l-1\}$.

Set $m(\mathbf{n}, \mathbf{d})=\sum_{i<j} n_{i} n_{j}\left[\left(\frac{d_{j}}{n_{j}}-\frac{d_{i}}{n_{i}}\right)+g-1\right]$. If $\mathbf{n}^{\prime} \subset \mathbf{n}$ define $\tau_{\left(\mathbf{n}^{\prime}, \mathbf{d}^{\prime}\right)}^{\mathbf{n}}$ to be 1 if $\frac{d_{k_{i}+1}}{n_{k_{i}+1}}>\cdots>$ $\frac{d_{k_{i+1}}}{n_{k_{i+1}}}$ for each $i \in\{0, \cdots, l-1\}$ and 0 otherwise. Note that $\tau_{\mathbf{n}, \mathbf{d}}^{n}=1$ if and only if $(\mathbf{n}, \mathbf{d})$ is a Shatz polygon.

Then given $M$, a $\mathcal{K}$-module, complete with the $\mathbb{1}(-1)$-adic topology, we have

Theorem 4.10. ([28], 2.4) For each function $f: \mathcal{P}_{n} \rightarrow M$ there is a unique function $\widehat{f}:$ $\mathcal{P}_{n, d} \rightarrow M$ such that

$$
f(\mathbf{n})=\sum_{\left(\mathbf{n}^{\prime} \mathbf{d}^{\prime}\right) \subset(\mathbf{n}, \mathbf{d})} \tau_{\left(\mathbf{n}^{\prime}, \mathbf{d}^{\prime}\right)}^{\mathbf{n}} \widehat{f}\left(\mathbf{n}^{\prime}, \mathbf{d}^{\prime}\right) \mathbb{1}\left(m(\mathbf{n}, \mathbf{d})-m\left(\mathbf{n}^{\prime}, \mathbf{d}^{\prime}\right)\right)
$$

for any $\mathbf{d}$. The value of $\widehat{f}$ on the maximal element of $\mathcal{P}_{n, d},(n, d)$, is given by

$$
\begin{aligned}
& \sum_{\mathbf{n} \in \mathcal{P}_{n}}(-1)^{\ell(\mathbf{n})-1} f(\mathbf{n})\left(\prod_{j=1}^{\ell(\mathbf{n})} \frac{1}{1-\mathbb{1}\left(-n_{j}-n_{j+1}\right)}\right) . \\
& \cdot \mathbb{1}\left(-\sum_{i<j} n_{i} n_{j}(g-1)-\sum_{j=1}^{\ell(\mathbf{n})-1}\left(n_{j}+n_{j+1}\right)\left\langle-\frac{n_{1}+\cdots+n_{j}}{n} d\right\rangle\right) .
\end{aligned}
$$

Theorem 4.11. If $n$ and $d$ are coprime, the motivic Poincaré polynomial of $N_{C}(n, d)$ is

$$
\begin{aligned}
& \sum_{s=1}^{n} \sum_{\substack{n_{1}+\cdots+n_{s}=n \\
n_{i} \in \mathbb{N}}}(-1)^{s-1} \frac{\left((\mathbb{1}+\mathbb{1})^{h^{1} C}\right)^{s}}{(\mathbb{1}-\mathbb{1}(-1))^{s-1}} \prod_{j=1}^{s} \prod_{i=1}^{n_{j}-1} \frac{(\mathbb{1}+\mathbb{1}(-i))^{h^{1} C}}{(\mathbb{1}-\mathbb{1}(-i))(\mathbb{1}-\mathbb{1}(-i-1))} \cdot \\
& \cdot \prod_{j=1}^{s-1} \frac{\mathbb{1}}{\mathbb{1}-\mathbb{1}\left(-n_{j}-n_{j+1}\right)} \cdot\left(-\sum_{i<j} n_{i} n_{j}(g-1)-\sum_{i=1}^{s-1}\left(n_{i}+n_{i+1}\right)\left\langle-\frac{n_{1}+\cdots+n_{j}}{n} d\right\rangle\right) .
\end{aligned}
$$


Proof of theorem 4.11. Apply the previous theorem to the $\mathcal{K}$-module $\mathcal{K}$. If $\mathbf{n}=\left(n_{1}, \cdots, n_{l}\right)$, choose any $d_{i} \in \mathbb{Z}$ and set

$$
f(\mathbf{n})=\prod_{i=1}^{l} \chi Q_{n_{i}, d_{i}}
$$

which is independent of the $d_{i}$.

Then theorem 4.10 combined with proposition 4.9 and proposition 4.4 .1 yield the following expression for $\chi Q_{n, d}^{s s}$

$$
\begin{aligned}
& \sum_{\left(n_{1}, \cdots, n_{s}\right) \in \mathcal{P}_{n}}(-1)^{s-1} \prod_{i=1}^{s}\left(\frac{(\mathbb{1}+\mathbb{1})^{h^{1} C}}{\left(\mathbb{1}-\mathbb{1}\left(-n_{i}\right)\right)} \prod_{j=1}^{n_{i}} \frac{(\mathbb{1}(-i)+\mathbb{1})^{h^{1} C}}{(\mathbb{1}-\mathbb{1}(-i))^{2}}\right) . \\
& \cdot\left(\prod_{i=1}^{s-1} \frac{1}{1-11\left(-n_{i}-n_{i+1}\right)}\right)\left(-\sum_{i<j} n_{i} n_{j}(g-1)-\sum_{i=1}^{s-1}\left(n_{i}+n_{i+1}\right)\left\langle-\frac{n_{1}+\cdots+n_{i}}{n} d\right\rangle\right)
\end{aligned}
$$

If $n$ and $d$ are coprime, dividing by $\frac{1}{1-1(-1)}$ yields the formula for $\chi N_{C}(n, d)$ in the statement of the theorem.

\section{Applications}

5.1. Hodge theory. In this subsection we assume the base field to be embeddable in the complex numbers.

Corollary 5.1. The Poincaré-Hodge polynomial of $N_{C}(n, d)$ is

$$
\begin{aligned}
& \sum_{n_{1}+\cdots+n_{\ell}=n}(-1)^{\ell-1} \frac{\left((1+x)^{g}(1+y)^{g}\right)^{\ell}}{(1-x y)^{\ell-1}} \prod_{j=1}^{\ell} \prod_{i=1}^{n_{j}-1} \frac{\left(1+x^{i} y^{i+1}\right)^{g}\left(1+x^{i+1} y^{i}\right)^{g}}{\left(1-x^{i} y^{i}\right)\left(1-x^{i+1} y^{i+1}\right)} . \\
& \cdot \prod_{j=1}^{\ell-1} \frac{1}{1-(x y)^{n_{j}+n_{j+1}}} \cdot(x y)^{\sum_{i<j} n_{i} n_{j}(g-1)+\sum_{i=1}^{\ell-1}\left(n_{i}+n_{i+1}\right)\left\langle-\frac{n_{1}+\cdots+n_{j}}{n} d\right\rangle} .
\end{aligned}
$$

Proof. We apply the morphism $P_{x y}: \mathcal{K} \longrightarrow \mathbb{Z}[[x, y]]$ to the expression in Theorem 4.11. As $P_{x y}$ is a ring morphism we just need to evaluate $P_{x y}(\mathbb{L})$ and $P_{x y}\left(\left(\mathbb{1}+\mathbb{L}^{n}\right)^{h^{1}(C)}\right)$. Clearly $P_{x y}(\mathbb{L})=x y$. From definition 2.3 and the additivity of $P_{x y}$

$$
P_{x y}\left(\left(1+\mathbb{L}^{n}\right)^{h^{1}(C)}\right)=\sum_{i} P_{x y}\left(\lambda^{i} h^{1}(C)\right)\left(x^{n} y^{n}\right)^{i} .
$$

If we replace the $x^{n} y^{n}$ coming from $\mathbb{L}^{n}$ by an indeterminate $T$ we have

$$
\begin{aligned}
\sum_{i} P_{x y}\left(\lambda^{i} h^{1} C\right) \cdot T^{i} & =\sum_{i} \sum_{p+q=i} \operatorname{dim} H^{p, q}(\operatorname{Jac} C, \mathbb{C}) x^{p} y^{q} \cdot T^{i} \\
& =P_{T x, T y}(\operatorname{Jac} C)=(1+T x)^{g}(1+T y)^{g} .
\end{aligned}
$$

Therefore we have shown that

$$
P_{x y}\left(1+\mathbb{L}^{n}\right)^{h^{1} C}=\left(1+x^{n+1} y^{n}\right)^{g}\left(1+x^{n} y^{n+1}\right)^{g} .
$$

This proves the corollary.

Let $\mathcal{L}$ be a line bundle of degree $d$ and note $N_{C}(n, \mathcal{L})$ the moduli space of stable vector bundles over $C$ of rank $n$ and determinant isomorphic to $\mathcal{L}$. We can recover the following result of Narasimhan and Ramanan from the previous corollary. 
Corollary 5.2. ([32]) The $\chi_{y^{-}}$genus of $N_{C}(n, \mathcal{L})$ is given by

$$
\left(\frac{1+(-1)^{n-1} y^{n}}{1-y} \prod_{i=1}^{n-1}\left(1+y^{i}\right)^{2}\right)^{g-1}
$$

In particular, both the signature and Euler characteristic of $N_{C}(n, \mathcal{L})$ are zero.

We now consider the category of absolute Hodge motives as defined in [13]. We note $\mathcal{M}_{k}^{A H}$ the category of absolute Hodge motives and $h_{A H}: \mathcal{V}_{k} \longrightarrow \mathcal{M}_{k}^{A H}$ the motivic functor.

Corollary 5.3. ([6], [7]) Let $\sigma$ be an embedding of an algebraically closed field $k$ in $\mathbb{C}$, then a $\sigma$-Hodge cycle on $N_{C}(n, d)$ is an absolute Hodge cycle.

Proof. By Theorem 4.5 $h_{A H}\left(N_{C}(n, d)\right)$ lies in the tannakian category generated by $h_{A H}(C)$.

The result is then a consequence of Theorem 6.25 in [13].

5.2. Finite fields. Let $C$ be a curve defined over the finite field with $q$ elements, $\mathbb{F}_{q}$.

Corollary 5.4. The number of points of $N_{C}(n, d)$ over a finite field of $q$ elements is given by

$$
\begin{aligned}
& \sum_{s=1}^{n} \sum_{\substack{n_{1}+\cdots+n_{s}=n \\
n_{i} \in \mathbb{N}^{*}}}(-1)^{s-1} \frac{\left(\# J a c(C)\left(\mathbb{F}_{q}\right)\right)^{s}}{(1-q)^{s-1}} \prod_{j=1}^{s} \prod_{i=1}^{n_{j}-1} \zeta_{C}(-i) \cdot \\
& \cdot \prod_{j=1}^{s-1} \frac{1}{1-q^{n_{j}+n_{j+1}}} \cdot q^{\sum_{i<j} n_{i} n_{j}(g-1)+\sum_{i=1}^{s-1}\left(n_{i}+n_{i+1}\right)\left\langle-\frac{n_{1}+\cdots+n_{j}}{n} d\right\rangle .}
\end{aligned}
$$

Proof. By a result of Grothendieck ([19]) there exists a smooth projective scheme, $\mathcal{C}$, over the spectrum of a complete local ring of characteristic zero with residue field $\mathbb{F}_{q}, S$, whose special fibre is isomorphic to $C$. We shall note the generic fibre of this scheme by $C_{0}$.

By Theorem 1.1 one can construct a scheme $N_{\mathcal{C}}(n, d)$ smooth and projective over with generic fibre $N_{C_{0}}(n, d)$ together with a morphism $N_{\mathcal{C}}(n, d) \times_{S} \operatorname{Spec}\left(\mathbb{F}_{q}\right) \longrightarrow N_{C}(n, d)$ that induces a bijection on the sets of $\mathbb{F}_{q}$-points. We write $N^{\prime}=N_{\mathcal{C}}(n, d) \times_{S} \operatorname{Spec}\left(\mathbb{F}_{q}\right)$.

Fix a prime $\ell$ not dividing $q$. The base change theorems shows that there is a natural isomorphism $H^{*}\left(N^{\prime} \otimes_{\mathbb{F}_{q}} \overline{\mathbb{F}}_{q}, \mathbb{Q}_{\ell}\right) \simeq H^{*}\left(N_{C_{0}}(n, d) \otimes_{K} \bar{K}, \mathbb{Q}_{\ell}\right)$ equivariant with respect to the actions of the Galois groups. In particular $\# N_{C}(n, d)\left(\mathbb{F}_{q}\right)=\# N^{\prime}\left(\mathbb{F}_{q}\right)=\nu_{q}^{1}\left(\chi\left(N^{\prime}\right)\right)$ is equal to $\nu_{q}^{1}\left(\chi\left(N_{C_{0}}(n, d)\right)\right)$ which can be computed with the aid of Theorem 4.11.

Given that $\nu_{q}^{t}: \mathcal{K} \longrightarrow \mathbb{Q}_{\ell}[[t]]$ is a ring morphism, we see from the expression in Theorem 4.11 that it is only necessary to evaluate $\nu_{q}^{t}$ on $\mathbb{L}$ and $(\mathbb{1}+\mathbb{L})^{h^{1}\left(C_{0}\right)}$. Clearly $\nu_{q}^{t}(\mathbb{L})=\operatorname{Tr}\left(\left.\operatorname{Fr}\right|_{\mathbb{Q}_{\ell}(-1)}\right)=$ $q t^{2}$.

Call $\omega_{1}, \ldots, \omega_{2 g}$ be the eigenvalues of the geometric Frobenius morphism acting on $H^{1}\left(C \otimes_{\mathbb{F}_{q}}\right.$ $\left.\overline{\mathbb{F}}_{q}, \mathbb{Q}_{\ell}\right)$ and set $P_{1}(t)=\prod_{t=1}^{2 g}\left(1-\omega_{i} t\right)$. Then we have

$$
\begin{aligned}
\nu_{q}^{t}\left(\mathbb{1}+\mathbb{L}^{n}\right)^{h^{1} C_{0}} & =\sum_{i} \operatorname{Tr}\left(\left.\operatorname{Fr}\right|_{\wedge^{i} H^{1}\left(\bar{C}, \mathbb{Q}_{\ell}\right)(-n i)}\right) t^{i+2 n i} \\
& =\sum_{i} \sum_{j_{1}<\cdots<j_{i}} \omega_{j_{1}} \cdots \omega_{j_{i}} q^{n i} t^{(2 n+1) i}=P_{1}\left(q^{n} t^{2 n+1}\right)
\end{aligned}
$$

The result now follows by recalling that $\zeta_{C}(-s)=\frac{P_{1}\left(q^{s}\right)}{\left(1-q^{s}\right)\left(1-q^{s+1}\right)}$.

\subsection{On some conjectures.}


5.3.1. Hodge and Tate conjectures. Recall that in each cohomology theory there are cycle class morphisms $c l_{i}: C H^{i}(X) \longrightarrow H^{2 i}(X)$ for each $X \in \mathrm{Ob} \mathcal{V}_{k}$. These can be extended to Chow motives.

Definition 5.5. 1. Define the function $P_{A}: K_{0} \mathcal{M}_{k}^{+} \longrightarrow \mathbb{Z}[t]$ by $P_{A}([M], t)=\sum_{i} \operatorname{dim} \operatorname{Im}$ $\left(c l_{i}: C H^{i}(M) \longrightarrow H^{2 i}(M)\right) \cdot t^{i}$ for $M$ an effective motive.

2. If $k=\mathbb{C}$ and $H^{*}$ singular cohomology define the function $P_{H}: K_{0} \mathcal{M}_{k}^{+} \longrightarrow \mathbb{Z}[t]$ by $P_{H}([M], t)=\sum_{i} \operatorname{dim}\left(H^{2 i}(M, \mathbb{Q}) \cap H^{i, i}(M, \mathbb{C})\right) \cdot t^{i}$ for $M$ an effective motive.

3. If $k$ is finitely generated over $\mathbb{Q}$ and $H^{*}$ is $\ell$-adic cohomology define the function $P_{T}$ : $K_{0} \mathcal{M}_{k}^{+} \longrightarrow \mathbb{Z}[t]$ by $P_{T}([M], t)=\sum_{i} \operatorname{dim}\left(H^{2 i}(M)(i)^{\operatorname{Gal}(\bar{k} \mid k)}\right) \cdot t^{i}$ for $M$ an effective motive.

It is immediate that for $? \in\{A, H, T\} P_{?}(\cdot, t)$ is an additive function and that for every integer $k$ we have $P_{?}(m(-k), t)=P_{?}(m, t) \cdot t^{k}$. This allows one to extent it to a function on $\mathcal{K}$.

The Hodge (resp. Tate) conjecture for a variety $X$ is equivalent to the equality $P_{A}(\chi(X), t)=$ $P_{H}(\chi(X), t)$ (resp. $\left.P_{A}(\chi(X), t)=P_{T}(\chi(X), t)\right)$. In more generality one can state the Hodge or Tate conjecture for motives or elements of $\mathcal{K}$ as follows:

Definition 5.6. We say the Hodge (resp. Tate) conjecture holds for $m \in \mathcal{K}$ if $P_{A}(m, t)=$ $P_{H}(m, t)$ (resp. $\left.P_{A}(m, t)=P_{T}(m, t)\right)$. We say either of these conjectures holds for $M \in O b \mathcal{M}_{k}^{+}$ whenever it does for $[m]$.

Remark 5.7. Let $M$ and $N$ be effective motives and $n \in \mathbb{N}$. It is easy to prove that the Hodge (resp. Tate) conjecture holds for $M$ and $N$ (resp. for $M$ ) if and only if it holds for $M \oplus N$ (resp. $M(-n))$.

Our main result here is the following elementary propagation principle.

Theorem 5.8. The elements of $\mathcal{K}$ that verify the Hodge (resp. Tate) conjecture are a sub$\mathbb{Z}[[\mathbb{L}]]$-module.

Proof. Clearly the elements of $\mathcal{K}$ that verify the Hodge (resp. Tate) conjecture are the kernel of the map

$$
P_{A}(\cdot, t)-P_{H}(\cdot, t): \mathcal{K} \longrightarrow \mathbb{Z}[[t]]
$$

(resp. $\left.P_{A}(\cdot, t)-P_{T}(\cdot, t)\right)$. As we have remarked, this is an additive map. It is also a map of $\mathbb{Z}[[\mathbb{L}]]$ modules if we consider $\mathbb{Z}[[t]]$ as a $\mathbb{Z}[[\mathbb{L}]]$-module via the natural isomorphism $\mathbb{Z}[[\mathbb{L}]] \longrightarrow \mathbb{Z}[[t]]$. The result follows

The following corollary shows that the Hodge or Tate conjectures for the moduli spaces, $N_{C}(n, d)$, can be reduced to the study of these conjectures on certain abelian varieties.

Corollary 5.9. If the Hodge (resp. Tate) conjecture holds for the $n$-th power of the jacobian, $\operatorname{Jac}(C)^{n}$, then it also holds for $N_{C}(n, d)$.

Proof. By S̆hermenev and Künneman we know there is a decomposition of the form $h(\mathrm{Jac} C) \simeq$ $\oplus \lambda^{i}\left(h^{1}(C)\right)$. The Künneth formula yields

$$
h\left(\operatorname{Jac}(C)^{n}\right)=\bigoplus_{i_{1}, \ldots, i_{n}} \lambda^{i_{1}} h^{1}(C) \otimes \cdots \otimes \lambda^{i_{n}} h^{1}(C) .
$$

We are assuming the Hodge (resp. Tate) conjecture to hold for $\operatorname{Jac}(C)^{n}$ so by remark 5.7 the same assumption holds for the motives $\lambda^{i_{1}} h^{1}(C) \otimes \cdots \otimes \lambda^{i_{n}} h^{1}(C)$. But Theorem 4.11 states that $\chi\left(N_{C}(n, d)\right)$ lies in the $\mathbb{Z}[[\mathbb{L}]]$-module generated by these motives. The result now follows from the previous theorem. 
Given that the Hodge conjecture holds for the general jacobian we recover the following result of Biswas and Narasimhan:

Corollary 5.10. ([8]) The Hodge conjecture for $N_{C}(n, d)$ holds for the general curve.

5.3.2. Standard conjecture of Lefschetz type. In this subsection we shall use motives with respect to a given homological equivalence (see [36]), $\mathcal{M}_{k}^{\text {hom }}+$. From the definition of $\mathcal{M}_{k}^{\text {hom }+}$ it is immediate to see that the function $P_{A}$ defined above gives a function on $K_{0} \mathcal{M}_{k}^{\text {hom+ }}$ and on its completion along $\mathbb{L}$ which we shall note $\mathcal{K}^{\text {hom }}$.

We start by extending this conjecture to elements of $\mathcal{K}^{\text {hom }}$. The standard conjecture of Lefschetz type $A$ is equivalent to the equality of polynomials $P_{A}\left(\chi(X)^{\vee}, t^{-1}\right)=P_{A}(\chi(X), t)$. More generally we conjecture that for every $m \in K_{0} \mathcal{M}_{k}^{\text {hom+ }}$ :

$\mathbf{A}(\mathbf{m}): P_{A}\left(m^{\vee} ; t^{-1}\right)=P_{A}(m ; t)$.

Note that the function $f(m)=P_{A}\left(m^{\vee} ; t^{-1}\right)-P_{A}(m ; t)$ is additive and verifies $f(m(-k))=$ $f(m) \cdot t^{k}$. This serves to prove that for $m, n \in K_{0} \mathcal{M}_{k}^{\text {hom+ }}$ and $k \in \mathbb{N} A(m+n)$ (resp. $\left.A(m(k))\right)$ holds if $A(m)$ and $A(n)$ do (resp. $A(m)$ does). It also shows that the statement $\mathbf{A}(\mathbf{m})$ makes sense for $m \in \mathcal{K}^{\text {hom }}$.

Recall that the general standard conjecture of Lefschetz type implies that the Künneth components of the diagonal are algebraic. In other words $B(X) \Leftarrow C(X)$. Therefore for such a variety we have a unique decomposition $h(X)=\oplus h^{i}(X)$ descending to the usual one in cohomology.

Our main result here is the following propagation principle.

Theorem 5.11. Let $X_{1}, \ldots, X_{n} \in \mathrm{Ob} \mathcal{V}_{k}$ verify the standard conjecture of Lefschetz type $B$.

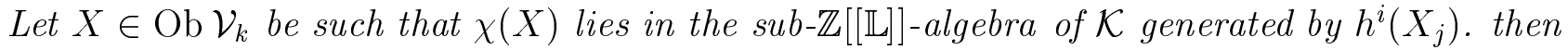
$B(X)$ holds.

Proof. By [25] we know that $A(X \times X) \Rightarrow B(X)$, therefore it is enough to see that for an $X$ as in the statement of the theorem , $A(X)$ holds. For such an $X$ we have that $\chi(X)$ is a sum of elements of the form

$$
a \cdot\left[h^{i_{1}}\left(X_{j_{1}}\right) \otimes \cdots \otimes h^{i_{r}}\left(X_{j_{r}}\right)\right](-m), \quad a \in \mathbb{Z}, \quad m \in \mathbb{N} .
$$

As we have remarked it is enough to prove that $A$ holds for motives of the form

$$
h^{i_{1}}\left(X_{j_{1}}\right) \otimes \cdots \otimes h^{i_{r}}\left(X_{j_{r}}\right) .
$$

Set $i=i_{1}+\cdots+i_{r}$. By lemma 5.12 we have an isomorphism of motives

$$
h^{i_{1}}\left(X_{j_{1}}\right) \otimes \cdots \otimes h^{i_{r}}\left(X_{j_{r}}\right) \simeq\left(h^{i_{1}}\left(X_{j_{1}}\right) \otimes \cdots \otimes h^{i_{r}}\left(X_{j_{r}}\right)\right)^{\vee}(-i) .
$$

Taking $A^{i / 2}$

$$
\begin{aligned}
A^{i / 2}\left(h^{i_{1}}\left(X_{j_{1}}\right) \otimes \cdots \otimes h^{i_{r}}\left(X_{j_{r}}\right)\right) & \simeq A^{i / 2}\left(\left(h^{i_{1}}\left(X_{j_{1}}\right) \otimes \cdots \otimes h^{i_{r}}\left(X_{j_{r}}\right)\right)^{\vee}(-i)\right) \\
& \simeq A^{-i / 2}\left(\left(h^{i_{1}}\left(X_{j_{1}}\right) \otimes \cdots \otimes h^{i_{r}}\left(X_{j_{r}}\right)\right)^{\vee}\right) .
\end{aligned}
$$

As $A^{i / 2}$ is the only nonzero cycle class space of (16) $A$ follows.

Lemma 5.12. Let $X$ verify the standard conjecture $B$. Then $h^{i}(X) \simeq h^{i}(X)^{\vee}(-i)$ for all $i$.

Proof. By taking into account the natural isomorphism

$$
h^{i}(X)^{\vee} \simeq h^{2 \operatorname{dim} X-i}(X)(-\operatorname{dim} X),
$$


we may assume that $i \leq \operatorname{dim} X$. Let $L$ be the Chern class of an ample line bundle on $X$. We claim that the morphism 'multiplication by $L^{\operatorname{dim} X-i}$,

$$
\overline{L^{\operatorname{dim} X-i}}: h^{i}(X) \longrightarrow h^{2 \operatorname{dim} X-i}(X)(\operatorname{dim} X-i) \simeq h^{i}(X)^{\vee}(-i)
$$

(see [36] 2.1 for the notation) is an isomorphism.

From the assumption on $X$ we have a morphism of motives

$$
\overline{\Lambda^{\operatorname{dim} X-i}}: h^{2 \operatorname{dim} X-i}(X)(\operatorname{dim} X-i) \longrightarrow h^{i}(X)
$$

The claim follows from the fact that the cohomology functor from motives with respect to homological equivalence to vector spaces is faithful. From standard Lefschetz theory $\Lambda^{2 \operatorname{dim} X-i}$ o $L^{2 \operatorname{dim} X-i}$ (resp. $L^{2 \operatorname{dim} X-i} \circ \Lambda^{2 \operatorname{dim} X-i}$ ) is the identity on $H^{i}(X)\left(\right.$ resp. on $\left.H^{2 \operatorname{dim} X-i}(X)\right)$, by faithfulness $\overline{\Lambda^{2 \operatorname{dim} X-i}} \circ \overline{L^{2 \operatorname{dim} X-i}}=I d$ and $\overline{L^{2 \operatorname{dim} X-i}} \circ \overline{\Lambda^{2 \operatorname{dim} X-i}}=I d$ so (17) is an isomorphism as claimed.

We can now prove that the standard conjecture of Lefschetz type $B$ holds for our moduli spaces. This has already been proved over the field of the complex numbers by I. Biswas and M.S. Narasimhan by monodromy techniques ([8]).

Corollary 5.13. The standard conjecture of Lefschetz type B holds for $N_{C}(n, d)$.

Proof. By theorem 4.11 the motivic Poincaré polynomial $\chi\left(N_{C}(n, d)\right)$ lies in the sub-ZZZ[ $\left.\left.\mathbb{L}\right]\right]$ algebra of $\mathcal{K}$ generated by $\lambda^{i} h^{1}(C)$ for $i \in\{0, \ldots, 2 g\}$.

The Corollary now follows from the previous Theorem taking in account that conjecture $B$ is true for $\operatorname{Jac}(C)$ as is shown in [25].

5.3.3. Semisimplicity of Galois actions. Let $k$ be finitely generated extension of $\mathbb{Q}$. The absolute Galois group $\operatorname{Gal}(\bar{k} \mid k)$ acts on the $\ell$-adic cohomology of any variety defined over $k$. In the case the variety is smooth and projective such action is conjectured to be semisimple by Grothendieck and Serre.

Proposition 5.14. For coprime $n$ and $d$ the action of the Galois group Gal $(\bar{k} \mid k)$ on $H^{*}\left(N_{C}(n, d) \otimes_{k}\right.$ $\left.\bar{k}, \mathbb{Q}_{\ell}\right)$ is semisimple.

Proof. Theorem 4.5 shows that $h\left(N_{C}(n, d)\right)$ lies in the tensor category generated by $h^{1}(C)$ and $\mathbb{L}$. This implies that the $\operatorname{Gal}(\bar{k} \mid k)$-module $H^{*}\left(N_{C}(n, d) \otimes_{k} \bar{k}, \mathbb{Q}_{\ell}\right)$ is a subobject of a sum of $\operatorname{Gal}(\bar{k} \mid k)$-modules of the type $H^{1}\left(C \otimes_{k} \bar{k}, \mathbb{Q}_{\ell}\right)^{\otimes n} \otimes \mathbb{Q}_{\ell}(-1)^{\otimes m}$ with $n, m \in \mathbb{N}$.

A result of Faltings ([15] Chapter IV) implies that the $\operatorname{Gal}(\bar{k} \mid k)$-module $H^{1}\left(C \otimes_{k} \bar{k}, \mathbb{Q}_{\ell}\right)$ is semisimple. The result follows.

\section{REFERENCES}

[1] M.F. Atiyah and R. Bott. The Yang-Mills equations over Riemann surfaces. Philos. Trans. Roy. Soc. London Ser., A308:523-615, 1982.

[2] A. Białynicki-Birula. Some theorems on actions of algebraic groups. Ann. of Math., 98:480-497, 1973.

[3] A. Białynicki-Birula. Some properties of the decompositions of algebraic varieties determined by actions of a torus. Bull. Acad. Polon. Sci., Ser. sci. math., astronom. et phys., 14:177-181, 1976.

[4] E. Bifet. Sur les points fixes du schema Quot $_{\mathcal{O}_{X}^{r}|X| k}$ sous l'action du tore $\mathbb{G}_{m, k}^{r}$. C. R. Acad. Sci. Paris, 309:609-612, 1989.

[5] E. Bifet, F. Ghione, and M. Letizia. On the Abel-Jacobi map for divisors of higher rank on a curve. Math. Ann., 299:641-672, 1994.

[6] I. Biswas. A remark on Hodge cycles on moduli spaces of rank 2 bundles over curves. J. reine angew. Math., 470:143-152, 1995.

[7] I. Biswas. A correspondence between the moduli spaces of vector bundles over a curve. preprint, 1996. 
[8] I. Biswas and M.S. Narasimhan. Hodge classes of moduli spaces of parabolic bundles over the general curve. J. Algebraic Geometry, pages 697-715, 1997.

[9] S. Bloch. Algebraic cycles and higher K-theory. Adv. Math., 61:267-304, 1986.

[10] S. Bloch. The moving lemma for higher Chow groups. J. Algebraic Geometry, 3:537-568, 1994.

[11] J. Burillo. El polinomio de Poincaré-Hodge de un producto simétrico de variedades kählerianas compactas. Collect. Math., 41:59-69, 1990.

[12] S. del Baño and V. Navarro Aznar. On the motive of a quotient variety. Coll. Math., 49:203-226, 1998.

[13] P. Deligne and J. Milne. Tannakian Categories. In Hodge Cycles, Motives and Shimura Varieties, volume 900 of Lecture Notes in Mathematics, chapter 2. Springer Verlag, 1982.

[14] V. Desale and S. Ramanan. Poincaré polynomials of the variety of stable bundles. Math. Ann., 216:233-244, 1975.

[15] G. Faltings and G. Wüstoltz. Rational points, volume E6 of Aspects of Mathematics. Vieweg, 1984.

[16] W. Fulton. Intersection Theory, volume 3 Folge, Band 2 of Ergebnisse der Mathematik und ihrer Grenzgebiete. Springer Verlag, 1984.

[17] E. Getzler. The mixed Hodge structures of configuration spaces. I. alg-geom/9510018, 1995.

[18] A. Grothendieck. Torsion homologique et sections rationelles. In Séminaire Chevalley, 1958.

[19] A. Grothendieck. Géométrie formelle et géométrie algébrique. In Séminaire Bourbaki, no 182, 1958/59.

[20] A. Grothendieck. Standard conjectures on algebraic cycles. In Bombay Coll. Alg. Geom. 1968, pages 193199, Oxford, 1969.

[21] F. Guillén Santos and V. Navarro Aznar. Un critère d'extension d'un foncteur défini sur les schémas lisses. alg-geom/9505008, 1995.

[22] G. Harder. Eine Bemerkung zu einer Arbeit von P.E. Newstead. Jour. für Math., 242:16-25, 1970.

[23] G. Harder and M.S. Narasimhan. On the cohomology groups of moduli spaces of vector bundles over curves. Math. Ann., 212:215-248, 1975.

[24] B. Iversen. A fixed point formula for actions of tori on algebraic varieties. Invent. Math., 16:229-236, 1972.

[25] S.L. Kleiman. Algebraic cycles and the Weil conjectures. In Dix esposés sur la cohomologie des schémas, pages 359-386. North Holland, Amsterdam, 1968.

[26] B. Köck. Chow motif and higher Chow theory of G/P. Manuscripta math., 70:363-372, 1991.

[27] K. Künnemann. On the Chow motive of an abelian scheme. In J.P. Serre U. Jannsen, S. Kleiman, editor, Motives, pages 189-205. Proc. Symp. Pure Math. 55, 1994.

[28] G. Laumon and M. Rapoport. The Langlands lemma and the Betti numbers of stacks of $G$-bundles on a curve. Int. J. of Math., 7:29-45, 1996.

[29] I.G. MacDonald. The Poincaré polynomial of a symmetric product. Proc. Camb. Phil. Soc., 58:563-568, 1962.

[30] Y. Manin. Correspondences, motives and monoidal transformations. Math. USSR Sb., 6:439-470, 1968.

[31] D. Mumford, J. Fogarty, and F. Kirwan. Geometric Invariant Theory, volume 34 of Ergebnisse der Mathematik und ihrer Grenzgebiete. Springer Verlag, 1992. Third edition.

[32] M.S. Narasimhan and S. Ramanan. Generalised Prym varieties as fixed points. Journal of the Indian Math. Soc., 39:1-19, 1975.

[33] P.E. Newstead. Introduction to moduli problems and orbit spaces. Tata Institute of Fundamental Research, Bombay, 1978.

[34] P.E. Newstead. Comparison theorems for conic bundles. Math. Proc. Camb. Phil. Soc., 90:21-31, 1981.

[35] D. Quillen. Higher algebraic K-theory I. In Algebraic K-theory, Lecture Notes in Mathematics 341. Springer Verlag, 1973.

[36] A.J. Scholl. Classical motives. In J.P. Serre U. Jannsen, S. Kleiman, editor, Motives, pages 163-187. Proc. Symp. Pure Math. 55, 1994.

[37] A.M. S̆hermenev. The motive of an abelian variety. Funct. Anal., 8:55-61, 1974.

MaX-Planck-Institut für Mathematik, Gotfried-Claren-Strasse 26, 53225 Bonn, GERMany 